\title{
COMPOSITION OPERATORS ON THE BLOCH SPACE OF THE UNIT BALL OF A HILBERT SPACE
}

\author{
OSCAR BLASCO*, PABLO GALINDO ${ }^{\dagger}$, MIKAEL LINDSTRÖM $^{\dagger}$, AND ALEJANDRO MIRALLES $^{\ddagger}$
}

\begin{abstract}
Every analytic self-map of the unit ball of a Hilbert space induces a bounded composition operator on the space of Bloch functions. Necessary and sufficient conditions for compactness of such composition operators are provided, as well as some examples that clarify the connections among such conditions.
\end{abstract}

\section{INTRODUCTION}

Let $E$ be a complex Hilbert space of arbitrary dimension and denote $B_{E}$ its open unit ball. The space $\mathcal{B}\left(B_{E}\right)$ of Bloch functions was introduced in [1]. There it was shown that it can be endowed with a (modulo the constant functions) norm that is invariant under the automorphisms of $B_{E}$; see section 3 below for the basics. This article studies composition operators acting on $\mathcal{B}\left(B_{E}\right)$, i.e., self-maps of $\mathcal{B}\left(B_{E}\right)$ defined according to $C_{\varphi}(f)=f \circ \varphi$, for a given analytic map $\varphi: B_{E} \rightarrow B_{E}$. As in the finite dimensional case, every composition operator is bounded, actually of norm not greater than 1 for the invariant norm if the symbol vanishes at 0 , and also the hyperbolic metric on $B_{E}$ measures the distance between evaluations in the dual space. We also study the compactness of composition operators, providing necessary and sufficient conditions. There are two common requirements for both the necessity and the sufficiency:

$$
\begin{aligned}
& \lim _{\|\varphi(z)\| \rightarrow 1} \frac{\left(1-\|z\|^{2}\right)\|\mathcal{R} \varphi(z)\|}{\sqrt{1-\|\varphi(z)\|^{2}}}=0 \quad \text { and } \\
& \lim _{\|\varphi(z)\| \rightarrow 1} \frac{\left(1-\|z\|^{2}\right)|\langle\varphi(z), \mathcal{R} \varphi(z)\rangle|}{1-\|\varphi(z)\|^{2}}=0
\end{aligned}
$$

where $R \varphi(z)$ denotes the radial derivative at $z$. The fact that for all $0<\delta<1, \varphi\left(\delta B_{E}\right)$ is relatively compact completes a necessary condition, while the additional assumption $\varphi\left(B_{E}\right) \cap$ $\delta B_{E}$ being relatively compact, provides a sufficient one. Such compactness requirements are trivially satisfied in the finite dimensional case, thus the two limits above yield an apparently new characterization.

Some of our techniques are inspired by J. Dai's paper [4]. However, there are some obstacles to avoid when allowing an infinite number of variables, like the lack of relative compactness of the ball, the number of components of the symbol or the use of the invariant Laplacian. And still a major one: uniform convergence on compact sets does not imply uniform convergence on compact sets of the derivatives; this only happens in the finite dimensional setting (see [3]). Such obstacle causes the lengthy proof of our main result Theorem 4.13. In the final section we present several examples that discuss the relations among the conditions we have found.

2010 Mathematics Subject Classification. Primary 30D45, 46E50. Secondary 46G20.

Key words and phrases. composition operator, Bloch function in the ball, infinite dimensional holomorphy.

*Partially supported by MTM2011-23164.

${ }^{\dagger}$ Partially supported by MEC2011-22457.

${ }^{\ddagger}$ Partially supported by MEC2011-22457 and P1-1B2014-35. 


\section{BACKGROUND}

Let $\left(e_{k}\right)_{k \in \Gamma}$ be an orthonormal basis of $E$ that we fix at once. Then every $z \in E$ can be written as $z=\sum_{k \in \Gamma} z_{k} e_{k}$ and we write $\bar{z}=\sum_{k \in \Gamma} \overline{z_{k}} e_{k}$.

Given an analytic function $\varphi: B_{E} \rightarrow B_{E}$ we write $\varphi(x)=\sum_{k \in \Gamma} \varphi_{k}(x) e_{k}, \varphi^{\prime}(x): E \rightarrow E$ its derivative at $x$, and $\mathcal{R} \varphi(x)=\varphi^{\prime}(x)(x)$ its radial derivative at $x$.

We shall denote by $\varphi_{a}$ the Möbius transforms for Hilbert spaces. For each $a \in B_{E}, \varphi_{a}$ : $B_{E} \rightarrow B_{E}$ is defined by

$$
\varphi_{a}(x)=\left(s_{a} Q_{a}+P_{a}\right)\left(m_{a}(x)\right)
$$

where $s_{a}=\sqrt{1-\|a\|^{2}}, m_{a}: B_{E} \rightarrow B_{E}$ is the analytic function

$$
m_{a}(x)=\frac{a-x}{1-\langle x, a\rangle}
$$

and $P_{a}=\frac{1}{\|a\|^{2}} a \otimes a$ where $u \otimes v(x)=\langle x, u\rangle v$ and $Q_{a}=I d-P_{a}$ are the orthogonal projection on the one dimensional subspace generated by $a$ and on its orthogonal complement respectively. Since $\varphi_{a} \circ \varphi_{a}(x)=x$ one has $\left(\varphi_{a}\right)^{-1}=\varphi_{a}$ and $\varphi_{a}^{\prime}(a)=\left(\varphi_{a}^{\prime}(0)\right)^{-1}$.

Actually (see for instance [1, Lemma 3.2])

$$
\varphi_{a}^{\prime}(0)=-s_{a}^{2} P_{a}-s_{a} Q_{a}
$$

and

$$
\varphi_{a}^{\prime}(a)=-\frac{1}{s_{a}^{2}} P_{a}-\frac{1}{s_{a}} Q_{a} .
$$

The pseudo-hyperbolic and hyperbolic metrics on $B_{E}$ are respectively defined by

$$
\rho_{E}(x, y):=\left\|\varphi_{x}(y)\right\| \text { and } \beta_{E}(x, y):=\frac{1}{2} \log \frac{1+\rho_{E}(x, y)}{1-\rho_{E}(x, y)} .
$$

It is known ([6] p. 99) that

$$
\left\|\varphi_{x}(y)\right\|^{2}=1-\frac{\left(1-\|x\|^{2}\right)\left(1-\|y\|^{2}\right)}{|1-\langle x, y\rangle|^{2}} .
$$

Also

$$
\rho_{E}(x, y)=\sup \left\{\rho(f(x), f(y)): f \in H^{\infty}\left(B_{E}\right),\|f\|_{\infty} \leq 1\right\}
$$

where $\rho$ is the pseudo-hyperbolic metric on the open unit disk $\mathbb{D}$ in the complex plane given by $\rho(z, w)=\left|\frac{z-w}{1-\bar{z} w}\right|$ and $H^{\infty}\left(B_{E}\right)$ denotes the Banach space of bounded analytic functions on $B_{E}$ endowed with the sup-norm.

Since $(s+t) /(1+s t)$ is an increasing function of $s$ and $t$ for $0 \leq s, t \leq 1$, the sharpened form of the triangle inequality for $\rho(z, w)$ easily yields the same inequality for $\rho_{E}(x, y)$,

$$
\rho_{E}(x, y) \leq \frac{\rho_{E}(x, u)+\rho_{E}(u, y)}{1+\rho_{E}(x, u) \rho_{E}(u, y)}, \quad x, u, y \in B_{E} .
$$

The following estimate holds (see [1], Lemma 4.1):

$$
\rho_{E}(x, y) \leq \frac{\|x-y\|}{|1-\langle x, y\rangle|}, \quad x, y \in B_{E} .
$$


The open unit ball of $H^{\infty}\left(B_{E}\right)$ is invariant under post-composition with conformal self-maps of $\mathbb{D}$. By composing $f$ with a conformal self-map of $\mathbb{D}$ that maps $f(y)$ to 0 , one obtains that

$$
\rho_{E}(x, y)=\sup \left\{|f(x)|: f \in H^{\infty}\left(B_{E}\right),\|f\|_{\infty} \leq 1, f(y)=0\right\} .
$$

Recall that if $f: B_{E} \rightarrow \mathbb{C}$ is analytic we have $f^{\prime}(x)(y)=\langle y, \overline{\nabla f(x)}\rangle$ and $\left(f \circ \varphi_{x}\right)^{\prime}(0)(y)=$ $\langle y, \bar{\nabla} f(x)\rangle$, where $\widetilde{\nabla} f(x)$ denotes the invariant gradient of $f$ at $x \in B_{E}$ given by

$$
\widetilde{\nabla} f(x)=\nabla\left(f \circ \varphi_{x}\right)(0)
$$

The following result gives an explicit formula to compute the invariant gradient. It is a modification of Lemma 3.5 in [1] in a form that fits our purposes.

Lemma 2.1. Let $f: B_{E} \rightarrow \mathbb{C}$ be an analytic function and $x \in B_{E}$. Then

$$
\|\widetilde{\nabla} f(x)\|=\sup _{w \neq 0} \frac{|\langle\nabla f(x), \bar{w}\rangle|\left(1-\|x\|^{2}\right)}{\sqrt{\left(1-\|x\|^{2}\right)\|w\|^{2}+|\langle w, x\rangle|^{2}}} .
$$

Proof. For the linear functional $w \in E \mapsto\left\langle\varphi_{x}^{\prime}(0)(w), \overline{\nabla f(x)}\right\rangle$ we have

$$
\|\widetilde{\nabla} f(x)\|=\sup _{w \neq 0} \frac{\left|\left\langle\varphi_{x}^{\prime}(0)(w), \overline{\nabla f(x)}\right\rangle\right|}{\|w\|}=\sup _{w \neq 0} \frac{\left|\left\langle\nabla f(x), \overline{\varphi_{x}^{\prime}(0)(w)}\right\rangle\right|}{\|w\|} .
$$

Now we can replace $w$ by $\varphi_{x}^{\prime}(0)^{-1}(w)$ in the above formula, so

$$
\|\widetilde{\nabla} f(x)\|=\sup _{w \neq 0} \frac{|\langle\nabla f(x), \bar{w}\rangle|}{\left\|\varphi_{x}^{\prime}(0)^{-1}(w)\right\|} .
$$

In the proof of Lemma 3.5 in [1] it is shown that

$$
\left\|\varphi_{x}(0)^{-1}(w)\right\|=\frac{\sqrt{\left(1-\|x\|^{2}\right)\|w\|^{2}+|\langle w, x\rangle|^{2}}}{1-\|x\|^{2}}
$$

so the statement follows.

Throughout the paper $\varphi: B_{E} \rightarrow B_{E}$ denotes an analytic map and given $y \in E \backslash\{0\}$ and $w \in E$ with $\|w\| \leq 1$ we write

$$
\varphi_{y, w}(\lambda)=\left\langle\varphi\left(\lambda \frac{y}{\|y\|}\right), \bar{w}\right\rangle, \quad|\lambda|<1 .
$$

The following version of Schwarz-Pick lemma will be needed in the sequel. The analogue of these results in several variables has been proved in [2].

Lemma 2.2. Let $\varphi: B_{E} \rightarrow B_{E}$ be an analytic map and $y \in B_{E}$. Then

$$
\begin{gathered}
|\langle\mathcal{R} \varphi(y), \varphi(y)\rangle| \leq\|y\|\|\varphi(y)\| \frac{1-\|\varphi(y)\|^{2}}{1-\|y\|^{2}}, \\
\frac{\left(1-\|y\|^{2}\right)}{\|y\|}\|\mathcal{R} \varphi(y)\|+\|\varphi(y)\|^{2}\left|\left\langle\frac{\mathcal{R} \varphi(y)}{\|\mathcal{R} \varphi(y)\|}, \frac{\varphi(y)}{\|\varphi(y)\|}\right\rangle\right|^{2} \leq 1 . \\
\|\mathcal{R} \varphi(y)\| \leq 2 \frac{\left(1-\|\varphi(y)\|^{2}\right)^{1 / 2}}{1-\|y\|^{2}} . \\
\text { Furthermore if } \varphi(0)=0, \text { then }\|\varphi(y)\| \leq\|y\| .
\end{gathered}
$$


Proof. Let us fix $y \in B_{E} \backslash\{0\}, \varphi(y) \neq 0$ and $w \in E$ with $\|w\| \leq 1$. We apply the classical Schwarz lemma to $\varphi_{y, w}$ and get for any $|\lambda|<1$ that

$$
\left|\varphi_{y, w}^{\prime}(\lambda)\right| \leq \frac{1-\left|\varphi_{y, w}(\lambda)\right|^{2}}{1-|\lambda|^{2}}
$$

Now if $\lambda \neq 0$ we have $\varphi_{y, w}^{\prime}(\lambda)=\frac{1}{\lambda}\left\langle\mathcal{R} \varphi\left(\lambda \frac{y}{\|y\|}\right), \bar{w}\right\rangle$. Hence, for $\lambda=\|y\|$, it follows that

$$
|\langle\mathcal{R} \varphi(y), \bar{w}\rangle| \leq\|y\| \frac{1-|\langle\varphi(y), \bar{w}\rangle|^{2}}{1-\|y\|^{2}} .
$$

This shows (2.10) and (2.11) by choosing $w=\frac{\overline{\varphi(y)}}{\|\varphi(y)\|}$ and $w=\frac{\overline{\mathcal{R} \varphi(z)}}{\|\mathcal{R} \varphi(z)\|}$ respectively.

To get (2.12) we use the estimate $|\langle\mathcal{R} \varphi(y), \bar{w}\rangle| \leq 2\|y\| \frac{1-|\langle\varphi(y), \bar{w}\rangle|}{1-\|y\|^{2}}$.

In particular, for any $\theta \in[-\pi, \pi)$ and $\|w\|=1$, we see that

$$
\begin{gathered}
\left|\left\langle\frac{\left(1-\|y\|^{2}\right.}{2\|y\|} \mathcal{R} \varphi(y)+e^{i \theta} \varphi(y), \bar{w}\right\rangle\right| \leq \frac{1-\|y\|^{2}}{2\|y\|}|\langle\mathcal{R} \varphi(y), \bar{w}\rangle|+|\langle\varphi(y), \bar{w}\rangle| \leq 1 . \\
\text { Hence, }\left\|\frac{1-\|y\|^{2}}{2\|y\|} \mathcal{R} \varphi(y)+e^{i \theta} \varphi(y)\right\| \leq 1 \text { for } \theta \in[-\pi, \pi) .
\end{gathered}
$$

Now integrating over $\theta$ we obtain $\frac{\left(1-\|y\|^{2}\right)^{2}}{4\|y\|^{2}}\|\mathcal{R} \varphi(y)\|^{2}+\|\varphi(y)\|^{2} \leq 1$.

In the case $\varphi(0)=0$ using $\varphi_{y, w}(0)=0$ and scalar Schwarz lemma we obtain

$$
\left|\varphi_{y, w}(\lambda)\right| \leq|\lambda|
$$

for all $y \in B_{E} \backslash\{0\}, \varphi(y) \neq 0$ and $w \in E$. This implies (2.13) choosing again $\lambda=\|y\|$ and $w=\frac{\overline{\varphi(y)}}{\|\varphi(y)\|}$. This completes the proof.

For background on analytic (or holomorphic) mappings on infinite dimensional complex spaces we refer to [3].

\section{The Bloch SPACE}

The classical Bloch space $\mathcal{B}$ is the space of analytic functions on the open unit disk, $f: \mathbb{D} \rightarrow$ $\mathbb{C}$, such that the semi-norm $\|f\|_{\mathcal{B}}=\sup _{z \in \mathbb{D}}\left(1-|z|^{2}\right)|f(z)|$ is bounded; it becomes a Banach space when endowed with the norm $\|f\|_{\text {Bloch }}=|f(0)|+\|f\|_{\mathcal{B}}$. See [10] for general background on the classical Bloch space. The Bloch space of functions defined on the finite dimensional Euclidean ball was introduced by R. Timoney in [8]. See [9] for further information.

A function $f: B_{E} \rightarrow \mathbb{C}$ is a Bloch function if

$$
\|f\|_{\mathcal{B}\left(B_{E}\right)}=\sup _{x \in B_{E}}\left(1-\|x\|^{2}\right)\left\|f^{\prime}(x)\right\|<\infty .
$$

The space of Bloch functions is denoted by $\mathcal{B}\left(B_{E}\right)$ and it has been studied in [1]. As in the finite dimensional case, the space $H^{\infty}\left(B_{E}\right)$ is strictly contained in $\mathcal{B}\left(B_{E}\right)$ (see [1, Corollary 4.3]) and the following inequality holds for any $f \in H^{\infty}\left(B_{E}\right)$ :

$$
\|f\|_{\mathcal{B}\left(B_{E}\right)} \leq\|f\|_{\infty} \text {. }
$$


An equivalent semi-norm for the space of Bloch functions is given by

$$
\|f\|_{i n v}=\sup _{x \in B_{E}}\|\widetilde{\nabla} f(x)\|<\infty .
$$

This semi-norm satisfies $\|f \circ \varphi\|_{\text {inv }}=\|f\|_{\text {inv }}$ for any $f \in \mathcal{B}\left(B_{E}\right)$ and any automorphism $\varphi$ of $B_{E}$. The space $\mathcal{B}\left(B_{E}\right)$ is usually endowed with the norm $\|f\|_{B l o c h\left(B_{E}\right)}=|f(0)|+\|f\|_{i n v}$ and then it becomes a Banach space.

Another equivalent semi-norm is given by

$$
\|f\|_{\text {rad }}=\sup _{x \in B_{E}}\left(1-\|x\|^{2}\right)|\mathcal{R} f(x)|
$$

where $\mathcal{R} f(x)=f^{\prime}(x)(x)$ is the radial derivative of $f$ at $x$.

We refer to [1, Thm 3.8] for all the equivalences of these semi-norms. In particular, we have the following inequalities:

$$
\|f\|_{\mathcal{B}\left(B_{E}\right)} \leq\|f\|_{\text {inv }} \leq\left(1+\frac{\sqrt{31}}{2}\right)\|f\|_{\mathcal{B}\left(B_{E}\right)} .
$$

The following result extends Theorem 5.5 in [10] to an infinite dimensional Hilbert space $E$ :

Theorem 3.1. Let $f: B_{E} \rightarrow \mathbb{C}$ be an analytic function. Then,

$$
\|f\|_{i n v}=\sup \left\{\frac{|f(x)-f(y)|}{\beta_{E}(x, y)}: x, y \in B_{E}, x \neq y\right\} .
$$

Proof. First we prove that

$$
\|f\|_{i n v} \geq M:=\sup \left\{\frac{|f(x)-f(y)|}{\beta_{E}(x, y)}: x, y \in B_{E}, x \neq y\right\} .
$$

If $\|f\|_{i n v}=\infty$, then we are done. So take $f \in \mathcal{B}\left(B_{E}\right)$ and $x, y \in B_{E}$. Then,

$$
\begin{aligned}
|f(x)-f(0)| & =\left|\left(\int_{0}^{1} f^{\prime}(x t) d t\right)(x)\right| \leq\|x\|\left\|\int_{0}^{1} \frac{f^{\prime}(x t)\left(1-\|x t\|^{2}\right)}{1-\|x t\|^{2}} d t\right\| \\
& \leq\|f\|_{\mathcal{B}\left(B_{E}\right)} \int_{0}^{1} \frac{\|x\|}{1-\|x\|^{2}|t|^{2}} d t=\|f\|_{\mathcal{B}\left(B_{E}\right)} \frac{1}{2} \log \frac{1+\|x\|}{1-\|x\|} .
\end{aligned}
$$

Consider $f \circ \varphi_{y} \in \mathcal{B}\left(B_{E}\right)$. By the inequality above, (3.2) and bearing in mind that $\|f \circ \varphi\|_{\text {inv }}=$ $\|f\|_{\text {inv }}$ for any automorphism $\varphi$, we have that

$$
\left|f \circ \varphi_{y}(z)-f \circ \varphi_{y}(0)\right| \leq\left\|f \circ \varphi_{y}\right\|_{i n v} \frac{1}{2} \log \frac{1+\|z\|}{1-\|z\|}=\|f\|_{i n v} \frac{1}{2} \log \frac{1+\|z\|}{1-\|z\|} .
$$

Selecting $z=\varphi_{y}(x)$, we have

$$
\begin{aligned}
|f(x)-f(y)| & \leq\left\|f \circ \varphi_{y}\right\|_{i n v} \frac{1}{2} \log \frac{1+\left\|\varphi_{y}(x)\right\|}{1-\left\|\varphi_{y}(x)\right\|} \\
& =\|f\|_{i n v} \frac{1}{2} \log \frac{1+\rho_{E}(x, y)}{1-\rho_{E}(x, y)}=\|f\|_{i n v} \beta_{E}(x, y) .
\end{aligned}
$$

Hence $\|f\|_{i n v} \geq M$.

Now we prove that $\|f\|_{i n v} \leq M$. Notice that

$$
|f(x)-f(0)| \leq M \beta_{E}(x, 0)=\frac{M}{2} \log \frac{1+\|x\|}{1-\|x\|},
$$


SO

$$
\frac{|f(x)-f(0)|}{\|x\|} \leq \frac{M}{2\|x\|} \log \frac{1+\|x\|}{1-\|x\|}
$$

for all $x \in B_{E} \backslash\{0\}$. For a unit vector $u \in E$, we consider the directional derivative $D_{u} f(0)$ given by

$$
D_{u} f(0)=\lim _{t \rightarrow 0} \frac{f(0+t u)-f(0)}{t}=\nabla f(0)(u) .
$$

If $x=t u$ and taking limits when $\|x\| \rightarrow 0$, we have that

$$
|\nabla f(0)(u)| \leq M \lim _{\|x\| \rightarrow 0} \frac{1}{2\|x\|} \log \frac{1+\|x\|}{1-\|x\|}=M
$$

since $\lim _{r \rightarrow 0} \frac{1}{r} \log \frac{1+r}{1-r}=2$, so $\|\nabla f(0)\| \leq M$. Notice that for any automorphism $\varphi$ on $B_{E}$, it is clear that

$$
M=\sup \left\{\frac{|(f \circ \varphi)(x)-(f \circ \varphi)(y)|}{\beta_{E}(x, y)}: x, y \in B_{E}, x \neq y\right\}
$$

since $\beta_{E}(\varphi(x), \varphi(y))=\beta(x, y)$. Hence for any $x \in B_{E}$ we have

$$
\|f\|_{i n v}=\sup _{x \in B_{E}}\left\|\nabla\left(f \circ \varphi_{x}\right)(0)\right\| \leq M
$$

and we are done.

Corollary 3.2. If $\delta_{x}(f)=f(x)$, we have that $\delta_{x} \in \mathcal{B}\left(B_{E}\right)^{*}$ and $\left\|\delta_{x}\right\| \leq L_{x}$ where

$$
L_{x}=\max \left\{\frac{1}{2} \log \frac{1+\|x\|}{1-\|x\|}, 1\right\} .
$$

Proof. From Theorem 3.1, we have for any $x \in B_{E}$

$$
\begin{gathered}
|f(x)-f(0)| \leq \frac{1}{2}\|f\|_{\mathcal{B}\left(B_{E}\right)} \log \frac{1+\|x\|}{1-\|x\|} \\
\text { Also }\left|\delta_{x}(f)\right| \leq|f(x)-f(0)|+|f(0)| \leq \frac{1}{2}\|f\|_{\mathcal{B}\left(B_{E}\right)} \log \frac{1+\|x\|}{1-\|x\|}+|f(0)| \\
\leq \max \left\{\frac{1}{2} \log \frac{1+\|x\|}{1-\|x\|}, 1\right\}\left(\|f\|_{\mathcal{B}\left(B_{E}\right)}+|f(0)|\right)=L_{x}\|f\|_{B \operatorname{loch}\left(B_{E}\right)} .
\end{gathered}
$$

Remark 3.3. For $x, y \in B_{E}$ we have

$$
\frac{1}{2}\|x-y\| \leq \rho_{E}(x, y) \leq\left\|\delta_{x}-\delta_{y}\right\| \leq \beta_{E}(x, y) .
$$

In particular, we observe that the norm topology of $\mathcal{B}\left(B_{E}\right)$ is finer than the compact open topology co.

As consequence of Theorem 3.1, we have that

Corollary 3.4. An analytic function $f: B_{E} \rightarrow \mathbb{C}$ belongs to $\mathcal{B}\left(B_{E}\right)$ if and only if there exists a constant $C>0$ such that

$$
|f(x)-f(y)| \leq C \beta_{E}(x, y) .
$$

Notice that the metric $\beta_{E}(x, y)$ can be also recovered from the Bloch semi-norm $\|f\|_{i n v}$ : 
Corollary 3.5. For any $x, y \in B_{E}$ we have

$$
\beta_{E}(x, y)=\sup \left\{|f(x)-f(y)|:\|f\|_{i n v} \leq 1\right\} .
$$

Proof. By Theorem 3.1 we have that $|f(x)-f(y)| \leq\|f\|_{\text {inv }} \beta_{E}(x, y)$ for all $f \in \mathcal{B}\left(B_{E}\right)$ and $x, y \in B_{E}$. Hence, $\sup \left\{|f(x)-f(y)|:\|f\|_{\text {inv }} \leq 1\right\} \leq \beta_{E}(x, y)$.

To check the other inequality follow the same pattern as Theorem 3.9 in [9] and recall [1, Lemma 3.3].

\section{Composition operators}

4.1. Boundedness. As it occurs in the finite dimensional case, every composition operator on $\mathcal{B}\left(B_{E}\right)$ is bounded.

Theorem 4.1. Every analytic map $\varphi: B_{E} \rightarrow B_{E}$ induces a bounded composition operator $C_{\varphi}: \mathcal{B}\left(B_{E}\right) \rightarrow \mathcal{B}\left(B_{E}\right)$.

Proof. Let $\varphi: B_{E} \rightarrow B_{E}$ be analytic and consider for any $f \in \mathcal{B}\left(B_{E}\right)$, the semi-norm $\|f \circ \varphi\|_{i n v}$. By Theorem 3.1, we have that

$$
\begin{aligned}
\|f \circ \varphi\|_{i n v} & =\sup \left\{\frac{|(f \circ \varphi)(x)-(f \circ \varphi)(y)|}{\beta_{E}(x, y)}: x, y \in B_{E}, x \neq y\right\} \\
& \leq \sup \left\{\frac{\mid(f(\varphi(x))-(f(\varphi(y)) \mid}{\beta_{E}(\varphi(x), \varphi(y))}: x, y \in B_{E}, \varphi(x) \neq \varphi(y)\right\},
\end{aligned}
$$

where last inequality holds because $\rho_{E}(x, y)$ is contractive for analytic maps $\varphi: B_{E} \rightarrow B_{E}$ and $h(t)=\frac{1}{2} \log \frac{1+t}{1-t}$ is non-decreasing. Since $\varphi\left(B_{E}\right) \subset B_{E}$, we get the estimate

$$
\|f \circ \varphi\|_{i n v} \leq \sup \left\{\frac{|f(x)-f(y)|}{\beta_{E}(x, y)}: x, y \in B_{E}, x \neq y\right\}=\|f\|_{i n v} .
$$

Further, using Corollary 3.2,

$$
\begin{gathered}
\left\|C_{\varphi}(f)\right\|_{B l o c h\left(B_{E}\right)}=\|f \circ \varphi\|_{i n v}+|f(\varphi(0))| \leq\|f\|_{i n v}+L_{\varphi(0)}\|f\|_{B l o c h\left(B_{E}\right)} \leq \\
\|f\|_{i n v}+|f(0)|+L_{\varphi(0)}\|f\|_{B \operatorname{Bloch}\left(B_{E}\right)}=\left(1+L_{\varphi(0)}\right)\|f\|_{\text {Bloch }\left(B_{E}\right)},
\end{gathered}
$$

and we conclude that $C_{\varphi}$ is bounded.

We provide another proof that relies on magnitudes that will appear further on.

Proof. Let $\|f\|_{\text {inv }} \leq 1$. Since $\mathcal{R}(f \circ \varphi)(z)=\langle\nabla f(\varphi(z)), \overline{\mathcal{R} \varphi(z)}\rangle$, we use Lemma 2.1 and obtain

$$
|\mathcal{R}(f \circ \varphi)(z)|^{2} \leq \| \widetilde{\nabla} f\left(\varphi(z) \|^{2} \frac{\left(1-\|\varphi(z)\|^{2}\right)\|\mathcal{R} \varphi(z)\|^{2}+|\langle\mathcal{R} \varphi(z), \varphi(z)\rangle|^{2}}{\left(1-\|\varphi(z)\|^{2}\right)^{2}} .\right.
$$

By combining this with Lemma 2.2 we conclude that

$$
|\mathcal{R}(f \circ \varphi)(z)|\left(1-\|z\|^{2}\right) \leq \sqrt{5} .
$$

Thus the boundedness of $C_{\varphi}$ is immediate if we assume $\varphi(0)=0$.

If $\varphi(0)=x \neq 0$, then we consider the mapping $\psi=\varphi_{x} \circ \varphi$, for which $\psi(0)=0$, and the bounded operator $C_{\psi}$. Since $\left\|f \circ \varphi_{x}\right\|_{i n v}=\|f\|_{i n v}$ it follows, using Corollary 3.2 as well, that $C_{\varphi_{x}}$ is continuous. Hence $C_{\varphi}=C_{\psi} \circ C_{\varphi_{x}}$ is continuous.

Remark 4.2. It is clear that if $\varphi(0)=0$, then $\left\|C_{\varphi}\right\| \leq 1$. 
4.2. Compactness. Now we proceed to discuss necessary and sufficient conditions for a composition operator on $\mathcal{B}\left(B_{E}\right)$ to be compact. We begin with some necessary ones.

4.2.1. Necessary conditions. The following result is a little improvement of a result due to Dai [4] for finitely many variables.

Lemma 4.3. For each $z \in B_{E}$ with $\varphi(z) \neq 0$, there is $\eta(z) \in E$, $\|\eta(z)\|=1$ with $\langle\varphi(z), \eta(z)\rangle=0$ such that for $\xi=\varphi(z)+\sqrt{1-\|\varphi(z)\|^{2}} \eta(z)$ one has

$$
|\langle\mathcal{R} \varphi(z), \xi\rangle| \geq \sqrt{1-\|\varphi(z)\|^{2}}\|\mathcal{R} \varphi(z)\|-\left(1+\frac{\sqrt{1-\|\varphi(z)\|^{2}}}{\|\varphi(z)\|}\right)|\langle\mathcal{R} \varphi(z), \varphi(z)\rangle| .
$$

Proof. We use the projection theorem for Hilbert spaces, so for each $z \in B_{E}$ with $\varphi(z) \neq 0$ there is $\eta(z) \in E,\|\eta(z)\|=1$ with $\langle\varphi(z), \eta(z)\rangle=0$ such that

$$
\mathcal{R} \varphi(z)=\alpha \frac{\varphi(z)}{\|\varphi(z)\|}+\beta \eta(z)
$$

where $\alpha=\frac{\langle\mathcal{R} \varphi(z), \varphi(z)\rangle}{\|\varphi(z)\|}$ and $\beta=\langle\mathcal{R} \varphi(z), \eta(z)\rangle$. Clearly $\|\xi\|=1,\langle\varphi(z), \xi\rangle=\|\varphi(z)\|^{2}$ and

$$
\begin{aligned}
&\langle\mathcal{R} \varphi(z), \xi\rangle=\langle\mathcal{R} \varphi(z), \varphi(z)\rangle+\sqrt{1-\|\varphi(z)\|^{2}} \beta \text {. Moreover, }|\alpha|^{2}+|\beta|^{2}=\|\mathcal{R} \varphi(z)\|^{2}, \text { so } \\
&|\langle\mathcal{R} \varphi(z), \xi\rangle| \geq \sqrt{1-\|\varphi(z)\|^{2}}|\beta|-|\langle\mathcal{R} \varphi(z), \varphi(z)\rangle| \\
& \geq \sqrt{1-\|\varphi(z)\|^{2}}|(|\mathcal{R} \varphi(z) \|-| \alpha \mid)-|\langle\mathcal{R} \varphi(z), \varphi(z)\rangle \mid \\
&=\sqrt{1-\|\varphi(z)\|^{2}}\|\mathcal{R} \varphi(z)\|-\left(1+\frac{\sqrt{1-\|\varphi(z)\|^{2}}}{\|\varphi(z)\|}\right)|\langle\mathcal{R} \varphi(z), \varphi(z)\rangle| .
\end{aligned}
$$

Lemma 4.4. The composition operator $C_{\varphi}: \mathcal{B}\left(B_{E}\right) \rightarrow \mathcal{B}\left(B_{E}\right)$ is compact if and only if for each bounded net $\left(f_{\alpha}\right)$ in $\mathcal{B}\left(B_{E}\right)$ such that $f_{\alpha} \rightarrow 0$ in $\left(\mathcal{B}\left(B_{E}\right)\right.$, co $)$ it follows that $\left\|C_{\varphi}\left(f_{\alpha}\right)\right\|_{\mathcal{B}\left(B_{E}\right)} \rightarrow 0$.

Proof. Suppose that $C_{\varphi}: \mathcal{B}\left(B_{E}\right) \rightarrow \mathcal{B}\left(B_{E}\right)$ is compact and let $\left(f_{\alpha}\right)$ be a bounded net in $\mathcal{B}\left(B_{E}\right)$ such that $f_{\alpha} \rightarrow 0$ in $\left(\mathcal{B}\left(B_{E}\right), c o\right)$. Then also $C_{\varphi}\left(f_{\alpha}\right) \rightarrow 0$ in $\left(\mathcal{B}\left(B_{E}\right), c o\right)$ and the norm closure of the set $\left\{C_{\varphi}\left(f_{\alpha}\right), 0\right\}$ is compact in $\mathcal{B}\left(B_{E}\right)$. Therefore $\left\|C_{\varphi}\left(f_{\alpha}\right)\right\|_{\mathcal{B}\left(B_{E}\right)} \rightarrow 0$.

If $C_{\varphi}$ is non-compact, then there are $\varepsilon>0$ and a sequence $\left(f_{n}\right)$ in $\mathcal{B}\left(B_{E}\right)$ such that $\left\|f_{n}\right\|_{\mathcal{B}\left(B_{E}\right)}=1$ and

$$
\left\|C_{\varphi}\left(f_{n}\right)-C_{\varphi}\left(f_{m}\right)\right\|_{\mathcal{B}\left(B_{E}\right)} \geq \varepsilon \text { for each } n \neq m .
$$

Now by Montel's theorem (see [3, Theorem 17.21]), there is a subnet $\left(f_{n(\alpha)}\right)$ of $\left(f_{n}\right)$ that converges uniformly on compact subsets of $B_{E}$ in $H\left(B_{E}\right)$. For each $n(\alpha)$, choose $n(\beta)>n(\alpha)$ such that $f_{n(\alpha)} \neq f_{n(\beta)}$ and let $g_{n(\alpha)}=f_{n(\alpha)}-f_{n(\beta)}$. Then $g_{n(\alpha)} \rightarrow 0$ in $\left(\mathcal{B}\left(B_{E}\right), c o\right)$, but $\left\|C_{\varphi}\left(g_{n(\alpha)}\right)\right\|_{\mathcal{B}\left(B_{E}\right)} \geq \varepsilon>0$.

Theorem 4.5. Assume that $C_{\varphi}: \mathcal{B}\left(B_{E}\right) \rightarrow \mathcal{B}\left(B_{E}\right)$ is a compact operator. Then

$$
\varphi\left(\delta B_{E}\right) \text { is relatively compact for each } 0<\delta<1 \text {, }
$$

$$
\lim _{\|\varphi(z)\| \rightarrow 1} \frac{\left(1-\|z\|^{2}\right)\|\mathcal{R} \varphi(z)\|}{\sqrt{1-\|\varphi(z)\|^{2}}}=0, \text { and }
$$




$$
\lim _{\|\varphi(z)\| \rightarrow 1} \frac{\left(1-\|z\|^{2}\right)|\langle\varphi(z), \mathcal{R} \varphi(z)\rangle|}{1-\|\varphi(z)\|^{2}}=0
$$

Proof. First we prove (4.1): Indeed, since the set $\left\{\delta_{z}:\|z\| \leq \delta\right\} \subset\left(\mathcal{B}\left(B_{E}\right)\right)^{*}$ is bounded and $C_{\varphi}^{*}$ is compact, $\left\{C_{\varphi}^{*}\left(\delta_{z}\right):\|z\| \leq \delta\right\}$ is relatively compact in $\mathcal{B}\left(B_{E}\right)^{*}$. The fact that $C_{\varphi}^{*}\left(\delta_{z}\right)=\delta_{\varphi(z)}$ allows us to conclude that $\varphi\left(\delta B_{E}\right)$ is relatively compact by appealing to (3.4).

Let $\left(n_{k}\right)$ be an increasing sequence in $\mathbb{N}$ and $\left(\xi_{k}\right)$ a sequence in $E$ with $\left\|\xi_{k}\right\| \leq 1$. According to [1, Corollary 4.3] the family $\left\{\langle z, \xi\rangle^{n_{k}}:\|\xi\|=1\right\}$ is bounded in $\mathcal{B}\left(B_{E}\right)$. Furthermore the resulting sequence $\left\{\left\langle z, \xi_{k}\right\rangle^{n_{k}}\right\}$ converges to zero in $\left(\mathcal{B}\left(B_{E}\right), c o\right)$ and therefore the compactness of $C_{\varphi}: \mathcal{B}\left(B_{E}\right) \rightarrow \mathcal{B}\left(B_{E}\right)$ implies, according to Lemma 4.4, that

$$
\lim _{k}\left\|\left\langle\varphi, \xi_{k}\right\rangle^{n_{k}}\right\|_{\text {rad }} \rightarrow 0, \text { when } k \rightarrow \infty \text {. }
$$

We have $\left\|\left\langle\varphi, \xi_{k}\right\rangle^{n_{k}}\right\|_{r a d}=\sup _{z \in B_{E}}\left(1-\|z\|^{2}\right) n_{k}\left|\left\langle\varphi(z), \xi_{k}\right\rangle\right|^{n_{k}-1}\left|\left\langle\mathcal{R} \varphi(z), \xi_{k}\right\rangle\right|$.

Let us first show (4.3). We suppose that there exist $\varepsilon>0$ and a sequence $\left(z_{k}\right) \in B_{E}$ such that $\left\|\varphi\left(z_{k}\right)\right\| \rightarrow 1$ and for each $k$,

$$
\frac{1-\left\|z_{k}\right\|^{2}}{1-\left\|\varphi\left(z_{k}\right)\right\|^{2}}\left|\left\langle\varphi\left(z_{k}\right), \mathcal{R} \varphi\left(z_{k}\right)\right\rangle\right| \geq \varepsilon
$$

Let $n_{k}$ be the integer part of $\frac{1}{1-\left\|\varphi\left(z_{k}\right)\right\|}$ and choose $\xi_{k}=\frac{\varphi\left(z_{k}\right)}{\left\|\varphi\left(z_{k}\right)\right\|}$. Since $\lim _{k}\left(1-\left\|\varphi\left(z_{k}\right)\right\|\right) n_{k}=$ 1 and $\lim _{k}\left\|\varphi\left(z_{k}\right)\right\|^{n_{k}-2}=\frac{1}{e}$, it follows from (4.4) that

$$
\begin{gathered}
0=\lim _{k \rightarrow \infty} \frac{1-\left\|z_{k}\right\|^{2}}{1-\left\|\varphi\left(z_{k}\right)\right\|^{2}}\left\|\varphi\left(z_{k}\right)\right\|^{n_{k}-2}\left|\left\langle\mathcal{R} \varphi\left(z_{k}\right), \varphi\left(z_{k}\right)\right\rangle\right| \\
=\frac{1}{e} \lim _{k \rightarrow \infty} \frac{1-\left\|z_{k}\right\|^{2}}{1-\left\|\varphi\left(z_{k}\right)\right\|^{2}}\left|\left\langle\mathcal{R} \varphi\left(z_{k}\right), \varphi\left(z_{k}\right)\right\rangle\right|,
\end{gathered}
$$

which gives a contradiction if (4.5) holds. Thus (4.3) holds.

Let us now show (4.2). As above we suppose that there exist $\varepsilon>0$ and a sequence $\left(z_{k}\right) \in B_{E}$ such that

$$
\frac{1-\left\|z_{k}\right\|^{2}}{\sqrt{1-\left\|\varphi\left(z_{k}\right)\right\|^{2}}}\left\|\mathcal{R} \varphi\left(z_{k}\right)\right\| \geq \varepsilon .
$$

Choosing now $n_{k}$ the integer part of $\frac{1}{1-\left\|\varphi\left(z_{k}\right)\right\|}$ and $\xi_{k}=\varphi\left(z_{k}\right)+\sqrt{1-\left\|\varphi\left(z_{k}\right)\right\|^{2}} \eta\left(z_{k}\right)$ with $\left\|\eta\left(z_{k}\right)\right\|=1$ and $\left\langle\varphi\left(z_{k}\right), \eta\left(z_{k}\right)\right\rangle=0$, then we obtain from (4.4)

$$
\begin{gathered}
0=\lim _{k \rightarrow \infty} \frac{1-\left\|z_{k}\right\|^{2}}{1-\left\|\varphi\left(z_{k}\right)\right\|^{2}}\left(\left\|\varphi\left(z_{k}\right)\right\|^{n_{k}-1}\right)^{2}\left|\left\langle\mathcal{R} \varphi\left(z_{k}\right), \xi_{k}\right\rangle\right| \\
=\frac{1}{e^{2}} \lim _{k \rightarrow \infty} \frac{1-\left\|z_{k}\right\|^{2}}{1-\left\|\varphi\left(z_{k}\right)\right\|^{2}}\left|\left\langle\mathcal{R} \varphi\left(z_{k}\right), \xi_{k}\right\rangle\right| .
\end{gathered}
$$

This together with condition (4.3) and Lemma 4.3 yields a contradiction to (4.6). So (4.2) holds.

Remark 4.6. Realize that conditions (4.2) and (4.3) hold trivially true in case $\varphi\left(B_{E}\right) \subset r B_{E}$ for some $0 \leq r<1$. 
Remark 4.7. Note that $\varphi(z)=z$ satisfies (4.2) and fails (4.3). Also observe that

$$
\frac{\left(1-\|z\|^{2}\right)\langle\mathcal{R} \varphi(z), \varphi(z)\rangle}{1-\|\varphi(z)\|^{2}}=\frac{\left(1-\|z\|^{2}\right)\|\mathcal{R} \varphi(z)\|}{\sqrt{1-\|\varphi(z)\|^{2}}} \frac{\left\langle\frac{\mathcal{R} \varphi(z)}{\|\mathcal{R} \varphi(z)\|}, \varphi(z)\right\rangle}{\sqrt{1-\|\varphi(z)\|^{2}}} .
$$

Hence (4.3) implies (4.2) if there exists $\delta>0$ such that

$$
\inf _{\|\varphi(z)\| \geq \delta} \frac{\left|\left\langle\frac{\mathcal{R} \varphi(z)}{\|\mathcal{R} \varphi(z)\|}, \varphi(z)\right\rangle\right|}{\sqrt{1-\|\varphi(z)\|^{2}}}>0 .
$$

Proposition 4.8. Let $\varphi: B_{E} \rightarrow B_{E}$ be analytic such that $C_{\varphi}: \mathcal{B}\left(B_{E}\right) \rightarrow \mathcal{B}\left(B_{E}\right)$ is a compact operator. Then $\{\mathcal{R} \varphi(z):\|z\| \leq \delta\}$ is relatively compact for all $\delta<1$.

Proof. For $z \in B_{E}$ and $w \in E$ we consider the linear functional $\lambda_{z, w}$ acting on $f \in \mathcal{B}\left(B_{E}\right)$ according to $\lambda_{z, w}(f)=f^{\prime}(z)(w)=\langle w, \overline{\nabla f(z)}\rangle$. It is continuous since $\left|\lambda_{z, w}(f)\right| \leq \frac{\|w\|}{1-\|z\|^{2}}\|f\|_{\mathcal{B}\left(B_{E}\right)}$. Realize that

$$
C_{\varphi}^{*}\left(\lambda_{z, w}\right)(f)=\lambda_{z, w}(f \circ \varphi)=\left\langle\varphi^{\prime}(z) w, \overline{\nabla f(\varphi(z))}\right\rangle,
$$

and thus that $C_{\varphi}^{*}\left(\lambda_{z, z}\right)=\lambda_{\varphi(z), \mathcal{R} \varphi(z)}$.

Notice that $\mathcal{R} \varphi\left(\delta B_{E}\right)$ is a bounded subset of $E$ by (2.12) in Lemma 2.2.

Since $C_{\varphi}^{*}$ is compact and $\sup \left\{\left\|\lambda_{z, z}\right\|:\|z\| \leq \delta\right\}<\infty$ then

$$
\left\{C_{\varphi}^{*}\left(\lambda_{z, z}\right):\|z\| \leq \delta\right\}=\left\{\lambda_{\varphi(z), \mathcal{R} \varphi(z)}:\|z\| \leq \delta\right\}
$$

is relatively compact in $\mathcal{B}\left(B_{E}\right)^{*}$. Now we conclude that $\mathcal{R} \varphi\left(\delta B_{E}\right)$ is relatively compact because for the function $e_{u}(z)=\langle z, u\rangle$, we have $\mathcal{R} C_{\varphi}\left(e_{u}\right)(z)=\langle\mathcal{R} \varphi(z), u\rangle=\lambda_{\varphi(z), \mathcal{R} \varphi(z)}\left(e_{u}\right)$ and hence

$$
\left\|\mathcal{R} \varphi(z)-\mathcal{R} \varphi\left(z^{\prime}\right)\right\|=\sup _{\|u\| \leq 1}\left|\left\langle\mathcal{R} \varphi(z)-\mathcal{R} \varphi\left(z^{\prime}\right), u\right\rangle\right| \leq\left\|\lambda_{\varphi(z), \mathcal{R} \varphi(z)}-\lambda_{\varphi\left(z^{\prime}\right), \mathcal{R} \varphi\left(z^{\prime}\right)}\right\| .
$$

There are also necessary conditions in terms of the components of the symbol $\varphi$. Recall that $\left(e_{k}\right)_{k \in \Gamma}$ is an orthonormal basis of $E$ and $\varphi=\sum_{k \in \Gamma} \varphi_{k}(x) e_{k}$. Here, $\varphi_{k}=\left\langle\varphi, e_{k}\right\rangle$.

Proposition 4.9. Assume that $C_{\varphi}: \mathcal{B}\left(B_{E}\right) \rightarrow \mathcal{B}\left(B_{E}\right)$ is a compact operator. Then

$$
C_{\varphi_{k, l}}: \mathcal{B} \rightarrow \mathcal{B} \text { is compact }
$$

for all $k, l \in \Gamma$, where $\varphi_{k, l}(\lambda):=\varphi_{k}\left(\lambda e_{l}\right), \lambda \in \mathbb{D}$. Also,

$$
\lim _{k \in \Gamma} \sup _{z \in B_{E}} \frac{\left(1-\|z\|^{2}\right)\left|\mathcal{R} \varphi_{k}(z)\right|}{1-\left|\varphi_{k}(z)\right|^{2}}=0 .
$$

In particular, $\lim _{k \in \Gamma}\left\|\varphi_{k}\right\|_{\mathcal{B}\left(B_{E}\right)}=0$. And further,

$$
\lim _{\left|\varphi_{n}(z)\right| \rightarrow 1} \frac{\left(1-\|z\|^{2}\right)\left|\mathcal{R} \varphi_{n}(z)\right|}{1-\left|\varphi_{n}(z)\right|^{2}}=0 \quad n \in \Gamma,
$$

Proof. Let $y \in E \backslash\{0\}$ and $\|\xi\| \leq 1$. We write $F^{\xi}(x)=F(\langle x, \bar{\xi}\rangle), x \in B_{E}$, for each $F \in \mathcal{H}(\mathbb{D})$, and $f_{y}(\lambda)=f\left(\lambda \frac{y}{\|y\|}\right), \lambda \in \mathbb{D}$, for each $f \in \mathcal{H}\left(B_{E}\right)$. 
Consider $F \in \mathcal{B}$. Since $\nabla F^{\xi}(x)=F^{\prime}(\langle x, \bar{\xi}\rangle) \xi$ then $F^{\xi} \in \mathcal{B}\left(B_{E}\right)$ and

$$
\left(1-\|x\|^{2}\right)\left\|\nabla F^{\xi}(x)\right\| \leq\|\xi\|\|F\|_{\mathcal{B}} \frac{1-\|x\|^{2}}{1-|\langle x, \bar{\xi}\rangle|^{2}} \leq\|F\|_{\mathcal{B}}
$$

Hence the operator $E_{\xi}: F \in \mathcal{B} \mapsto F^{\xi} \in \mathcal{B}\left(B_{E}\right)$ is continuous.

If $f \in \mathcal{B}\left(B_{E}\right)$ and $\|y\| \leq 1$ then it is an easy calculation that $f_{y} \in \mathcal{B}$ and $\left\|f_{y}\right\|_{\mathcal{B}} \leq\|f\|_{\mathcal{B}\left(B_{E}\right)}$. Hence the operator $R_{y}: f \in \mathcal{B}\left(B_{E}\right) \mapsto f_{y} \in \mathcal{B}$ is continuous. For each $y, \xi \in B_{E}$ and $F \in \mathcal{B}$ we can write

$$
\left(C_{\varphi}\left(F^{\xi}\right)\right)_{y}(\lambda)=F^{\xi}\left(\varphi\left(\lambda \frac{y}{\|y\|}\right)\right)=F\left(\left\langle\varphi\left(\lambda \frac{y}{\|y\|}\right), \bar{\xi}\right\rangle\right)=C_{\varphi_{y, \xi}}(F)(\lambda) .
$$

So $C_{\varphi_{y, \xi}}=R_{y} \circ C_{\varphi} \circ E_{\xi}$ is compact. Then (4.7) follows because $\varphi_{k, l}=\varphi_{e_{k}, e_{l}}$.

Let us now show (4.8). Given a weakly null net $\left(\xi_{k}\right)_{k \in \kappa} \in E$ with $\left\|\xi_{k}\right\| \leq 1$, we consider $f_{k}(z)=\log \left(\frac{1}{1-\left\langle z, \xi_{k}\right\rangle}\right)$. According to [1, Corollary 4.4], $f_{k} \in \mathcal{B}\left(B_{E}\right)$ and $\left\|f_{k}\right\|_{\mathcal{B}\left(B_{E}\right)} \leq$ $\left\|\log \left(\frac{1}{1-\lambda}\right)\right\|_{\mathcal{B}}$. Thus, the net $\left\{f_{k}: k \in \kappa\right\}$ is bounded on compact subsets in $B_{E}$, hence a co-relatively compact set by Montel's theorem. Since $\lim _{k \in \kappa} f_{k}(z)=0$, it follows that $\left\{f_{k}\right.$ : $k \in \kappa\}$ converges to zero uniformly on compact sets of $B_{E}$. Hence $\lim _{k \in \kappa}\left\|C_{\varphi}\left(f_{k}\right)\right\|_{\mathcal{B}\left(B_{E}\right)}=0$. Now notice that $\mathcal{R}\left(C_{\varphi}\left(f_{k}\right)\right)(z)=\frac{\left\langle\mathcal{R} \varphi(z), \xi_{k}\right\rangle}{1-\left\langle\varphi(z), \xi_{k}\right\rangle}$. Therefore

$$
\lim _{k \in \kappa} \sup _{\|z\|<1} \frac{\left(1-\|z\|^{2}\right)\left|\left\langle\mathcal{R} \varphi(z), \xi_{k}\right\rangle\right|}{\left|1-\left\langle\varphi(z), \xi_{k}\right\rangle\right|}=0 .
$$

Assume now that (4.8) does not hold. Then there exist $\varepsilon>0$, and a subnet $\left(n_{k}\right)$ such that for every $n_{k}$ there is $z_{k}$ with

$$
\frac{\left(1-\left\|z_{k}\right\|^{2}\right)\left|\mathcal{R} \varphi_{n_{k}}\left(z_{k}\right)\right|}{1-\left|\varphi_{n_{k}}\left(z_{k}\right)\right|^{2}} \geq \varepsilon .
$$

Selecting now $\xi_{k}=e_{n_{k}} \overline{\varphi_{n_{k}}\left(z_{k}\right)}$, we get a weakly null net for which thus (4.10) holds. Then

$$
\sup _{\|z\|<1} \frac{\left(1-\|z\|^{2}\right)\left|\mathcal{R} \varphi_{n_{k}}(z) \| \varphi_{n_{k}}\left(z_{k}\right)\right|}{\left|1-\varphi_{n_{k}}(z) \overline{\varphi_{n_{k}}\left(z_{k}\right)}\right|} \rightarrow 0, \quad k \rightarrow \infty,
$$

that contradicts (4.11).

Finally, we prove (4.9). Let $n \in \Gamma$ and assume that (4.9) does not hold, that is there is $\varepsilon>0$ and a sequence $\left(z_{l}\right)$ with $\lim _{l \rightarrow \infty}\left|\varphi_{n}\left(z_{l}\right)\right|=1$ and

$$
\frac{\left(1-\left\|z_{l}\right\|^{2}\right)\left|\mathcal{R} \varphi_{n}\left(z_{l}\right)\right|}{1-\left|\varphi_{n}\left(z_{l}\right)\right|^{2}} \geq \varepsilon .
$$

Let $F_{l}(\lambda)=\log \frac{1}{1-\lambda \overline{\varphi_{n}\left(z_{l}\right)}}$ and $g_{l}(x)=F_{l}\left(\left\langle x, e_{n}\right\rangle\right)=\log \frac{1}{1-\left\langle x, e_{n}\right\rangle \overline{\varphi_{n}\left(z_{l}\right)}}$.

We may assume that $\varphi_{n}\left(z_{l}\right)$ converges to some $w_{0},\left|w_{0}\right|=1$. This means that $\left(g_{l}\right)$ coconverges to $g_{0}(x)=F_{0}\left(\left\langle x, e_{n}\right\rangle\right)=\log \frac{1}{1-\left\langle x, e_{n}\right\rangle \overline{w_{0}}}$ where $F_{0}(\lambda)=\log \frac{1}{1-\lambda \overline{w_{0}}}$. Next, notice that $C_{\varphi}\left(g_{l}\right)(x)=F_{l}\left(\left\langle\varphi(x), e_{n}\right\rangle\right)=F_{l} \circ \varphi_{n}(x)$.

The compactness of $C_{\varphi}$ yields that $\lim _{l}\left\|C_{\varphi}\left(g_{l}\right)-C_{\varphi}\left(g_{0}\right)\right\|_{\text {rad }}=0$. However,

$$
\begin{aligned}
& \left\|C_{\varphi}\left(g_{l}\right)-C_{\varphi}\left(g_{0}\right)\right\|_{\text {rad }}=\left\|F_{l} \circ \varphi_{n}-F_{0} \circ \varphi_{n}\right\|_{\text {rad }}= \\
& \sup _{x \in B_{E}}\left(1-\|x\|^{2}\right)\left|\mathcal{R}\left(F_{l} \circ \varphi_{n}\right)(x)-\mathcal{R}\left(F_{0} \circ \varphi_{n}\right)(x)\right|=
\end{aligned}
$$


A contradiction.

$$
\begin{gathered}
\sup _{x \in B_{E}}\left(1-\|x\|^{2}\right)|| F_{l}^{\prime}\left(\varphi_{n}(x)\right) \mathcal{R} \varphi_{n}(x)-F_{0}^{\prime}\left(\varphi_{n}(x)\right) \mathcal{R} \varphi_{n}(x) \mid= \\
\sup _{x \in B_{E}}\left(1-\|x\|^{2}\right)\left|\mathcal{R} \varphi_{n}(x)\right|\left|F_{l}^{\prime}\left(\varphi_{n}(x)\right)-F_{0}^{\prime}\left(\varphi_{n}(x)\right)\right|= \\
\sup _{x \in B_{E}}\left(1-\|x\|^{2}\right)\left|\mathcal{R} \varphi_{n}(x)\right|\left|\frac{\overline{\varphi_{n}\left(z_{l}\right)}}{1-\overline{\varphi_{n}\left(z_{l}\right)} \varphi_{n}(x)}-\frac{\overline{w_{0}}}{1-\overline{w_{0}} \varphi_{n}(x)}\right| \geq \\
\left(1-\left\|z_{l}\right\|^{2}\right)\left|\mathcal{R} \varphi_{n}\left(z_{l}\right)\right|\left|\frac{\overline{\varphi_{n}\left(z_{l}\right)}}{1-\overline{\varphi_{n}\left(z_{l}\right)} \varphi_{n}\left(z_{l}\right)}-\frac{\overline{w_{0}}}{1-\overline{w_{0}} \varphi_{n}\left(z_{l}\right)}\right|= \\
\frac{\left(1-\left\|z_{l}\right\|^{2}\right)}{1-\left|\varphi_{n}\left(z_{l}\right)\right|^{2}}\left|\mathcal{R} \varphi_{n}\left(z_{l}\right)\right| \frac{\overline{\varphi_{n}\left(z_{l}\right)}-\overline{w_{0}}}{1-\varphi_{n}\left(z_{l}\right) \overline{w_{0}}} \mid \geq \varepsilon .
\end{gathered}
$$

\subsubsection{Compactness criteria.}

Lemma 4.10. Let $f: B_{E} \rightarrow \mathbb{C}$ be analytic and $x, y \in B_{E}$. Then

$$
\left(1-\|x\|^{2}\right) \mathcal{R} f(x)=\frac{-1}{2 \pi i} \int_{|\xi|=1} f\left(\varphi_{x}(\xi x)\right) \frac{d \xi}{\xi^{2}}
$$

Proof. Observe that since $\varphi_{x}$ is self-inverse, $f=\left(f \circ \varphi_{x}\right) \circ \varphi_{x}$, hence

$$
\begin{aligned}
\langle y, \overline{\nabla f(x)}\rangle & =f^{\prime}(x)(y)=\left(f \circ \varphi_{x}\right)^{\prime}(0) \circ\left(\varphi_{x}\right)^{\prime}(x)(y) \\
& =\left(f \circ \varphi_{x}\right)^{\prime}(0)\left[\left(-\frac{1}{s_{x}^{2}} P_{x}-\frac{1}{s_{x}} Q_{x}\right)(y)\right] \\
& =-\frac{1}{s_{x}^{2}}\left(f \circ \varphi_{x}\right)^{\prime}(0)\left[P_{x}(y)\right]-\frac{1}{s_{x}}\left(f \circ \varphi_{x}\right)^{\prime}(0)\left[Q_{x}(y)\right] \\
& =-\frac{1}{s_{x}^{2}}\left\langle P_{x}(y), \overline{\widetilde{\nabla} f(x)}\right\rangle-\frac{1}{s_{x}}\left\langle Q_{x}(y), \overline{\widetilde{\nabla} f(x)}\right\rangle \\
& =-\frac{1}{s_{x}^{2}}\left\langle P_{x}(y), \overline{\widetilde{\nabla} f(x)}\right\rangle-\frac{1}{s_{x}}\left\langle y-P_{x}(y), \overline{\widetilde{\nabla} f(x)}\right\rangle .
\end{aligned}
$$

and using that $P_{x}$ is self-adjoint,

$$
\begin{aligned}
& \langle y, \overline{\nabla f(x)}\rangle=-\frac{1}{s_{x}^{2}}\left\langle y, P_{x}(\overline{\widetilde{\nabla} f(x)})\right\rangle-\frac{1}{s_{x}}\langle y, \overline{\widetilde{\nabla} f(x)}\rangle+\frac{1}{s_{x}}\left\langle y, P_{x}(\overline{\widetilde{\nabla} f(x)})\right\rangle \\
& =\left(-\frac{1}{s_{x}^{2}}+\frac{1}{s_{x}}\right)\left\langle y, \frac{\langle\overline{\widetilde{\nabla} f(x)}, x\rangle}{\|x\|^{2}} x\right\rangle-\frac{1}{s_{x}}\langle y, \overline{\widetilde{\nabla} f(x)}\rangle \\
& =\left(-\frac{1}{s_{x}^{2}}+\frac{1}{s_{x}}\right) \frac{\langle x, \overline{\widetilde{\nabla} f(x)}\rangle}{\|x\|^{2}}\langle y, x\rangle-\frac{1}{s_{x}}\langle y, \overline{\widetilde{\nabla} f(x)}\rangle \text {. }
\end{aligned}
$$

By the Cauchy formula we have that

$$
\langle x, \overline{\widetilde{\nabla} f(x)}\rangle=\left(f \circ \varphi_{x}\right)^{\prime}(0)(x)=\frac{1}{2 \pi i} \int_{|\xi|=1} f \circ \varphi_{x}(\xi x) \frac{d \xi}{\xi^{2}} \text { and }
$$




$$
\langle y, \widetilde{\nabla} f(x)\rangle=\left(f \circ \varphi_{x}\right)^{\prime}(0)(y)=\frac{1}{2 \pi i} \int_{|\xi|=1} f \circ \varphi_{x}(\xi y) \frac{d \xi}{\xi^{2}} .
$$

Thus equality (4.14) becomes

$$
\begin{gathered}
s_{x}^{2}\langle y, \overline{\nabla f(x)}\rangle= \\
\left(s_{x}-1\right) \frac{1}{2 \pi i} \int_{|\xi|=1} f \circ \varphi_{x}(\xi x) \frac{d \xi}{\xi^{2}} \frac{y y, x\rangle}{\|x\|^{2}}-s_{x} \frac{1}{2 \pi i} \int_{|\xi|=1} f \circ \varphi_{x}(\xi y) \frac{d \xi}{\xi^{2}}
\end{gathered}
$$

and we conclude by taking $y=x$.

Remark 4.11. From (4.14) we deduce the following identity that might be of independent interest

$$
s_{x}^{2} \nabla f(x)+s_{x} \widetilde{\nabla} f(x)=\left(s_{x}-1\right) \frac{\langle\widetilde{\nabla} f(x), \bar{x}\rangle}{\|x\|^{2}} \bar{x} .
$$

Lemma 4.12. For every $0<\delta<1$, there exists $C_{\delta}>0$ such that

$$
\left|\langle y, \overline{\nabla f(x)}\rangle-\left\langle y^{\prime}, \overline{\nabla f\left(x^{\prime}\right)}\right\rangle\right| \leq C_{\delta}\|f\|_{\mathcal{B}}\left\|x-x^{\prime}\right\|
$$

whenever $x, x^{\prime} \in \delta B_{E}$ and $\|y\| \leq 1,\left\|y^{\prime}\right\| \leq 1$, and $f \in \mathcal{B}\left(B_{E}\right)$.

Proof. Let $0<\varepsilon<\frac{1-\delta}{2}$. Since $\max \left\{\|x+\varepsilon \xi y\|,\left\|x^{\prime}+\varepsilon \xi y^{\prime}\right\|:|\xi|=1\right\} \leq \frac{1+\delta}{2}$, we conclude by taking $u=0$ in (2.5) that

$$
\rho_{E}\left(x+\varepsilon \xi y, x^{\prime}+\varepsilon \xi y^{\prime}\right) \leq \frac{4(1+\delta)}{4+(1+\delta)^{2}}<1 .
$$

Since $\frac{1}{2} \log \frac{1+r}{1-r} \leq \frac{r}{1-r}$ for all $0<r<1$, we have

$$
\beta_{E}\left(x+\varepsilon \xi y, x^{\prime}+\varepsilon \xi y^{\prime}\right) \leq \frac{\rho_{E}\left(x+\varepsilon \xi y, x^{\prime}+\varepsilon \xi y^{\prime}\right)}{1-\frac{4(1+\delta)}{4+(1+\delta)^{2}}},
$$

so it follows that for some constant $C_{\delta}^{\prime}$ depending only on $\delta$,

$$
\beta_{E}\left(x+\varepsilon \xi y, x^{\prime}+\varepsilon \xi y^{\prime}\right) \leq C_{\delta}^{\prime} \rho_{E}\left(x+\varepsilon \xi y, x^{\prime}+\varepsilon \xi y^{\prime}\right) .
$$

Next, using Cauchy formula we have for $x, x^{\prime} \in \delta B_{E},\|y\| \leq 1,\left\|y^{\prime}\right\| \leq 1$,

$$
\langle y, \overline{\nabla f(x)}\rangle-\left\langle y^{\prime}, \overline{\nabla f\left(x^{\prime}\right)}\right\rangle=\frac{1}{2 \pi i} \int_{|\xi|=1} f(x+\varepsilon \xi y)-f\left(x^{\prime}+\varepsilon \xi y^{\prime}\right) \frac{d \xi}{\xi^{2}} .
$$

From this, Theorem 3.1 and the equivalence of the semi-norms, we get that for some constant $C>0$

$$
\begin{aligned}
\left|\langle y, \overline{\nabla f(x)}\rangle-\left\langle y^{\prime}, \overline{\nabla f\left(x^{\prime}\right)}\right\rangle\right| & \leq \int_{0}^{2 \pi}\left|f\left(x+\varepsilon e^{i t} y\right)-f\left(x^{\prime}+\varepsilon e^{i t} y^{\prime}\right)\right| \frac{d t}{2 \pi} \\
& \leq C\|f\|_{\mathcal{B}\left(B_{E}\right)} \int_{0}^{2 \pi} \beta_{E}\left(x+\varepsilon e^{i t} y, x^{\prime}+\varepsilon e^{i t} y^{\prime}\right) d \xi .
\end{aligned}
$$

Applying (2.6) we find a constant $C_{\delta}>0$ depending only on $\delta$ such that

$$
\begin{array}{r}
\left|\langle y, \overline{\nabla f(x)}\rangle-\left\langle y^{\prime}, \overline{\nabla f\left(x^{\prime}\right)}\right\rangle\right| \leq C \cdot C_{\delta}^{\prime}\|f\|_{\mathcal{B}\left(B_{E}\right)} \int_{0}^{2 \pi} \rho_{E}\left(x+\varepsilon e^{i t} y, x^{\prime}+\varepsilon e^{i t} y^{\prime}\right) \frac{d t}{2 \pi} \\
\leq C_{\delta}\|f\|_{\mathcal{B}\left(B_{E}\right)} \int_{0}^{2 \pi}\left\|\left(x+\varepsilon e^{i t} y\right)-\left(x^{\prime}+\varepsilon e^{i t} y^{\prime}\right)\right\| \frac{d t}{2 \pi} \leq C_{\delta}\|f\|_{\mathcal{B}\left(B_{E}\right)}\left(\left\|x-x^{\prime}\right\|+2 \varepsilon\right) .
\end{array}
$$


Now let $\varepsilon \rightarrow 0$.

Theorem 4.13. Let $\varphi: B_{E} \rightarrow B_{E}$ be analytic. Assume that

(i) $\{\varphi(z):\|\varphi(z)\| \leq \delta\}$ is relatively compact for all $0<\delta<1$,

(ii) $\lim _{\|\varphi(z)\| \rightarrow 1} \frac{\left(1-\|z\|^{2}\right)\|\mathcal{R} \varphi(z)\|}{\sqrt{1-\|\varphi(z)\|^{2}}}=0$ and

(iii) $\lim _{\|\varphi(z)\| \rightarrow 1} \frac{\left(1-\|z\|^{2}\right)|\langle\varphi(z), \mathcal{R} \varphi(z)\rangle|}{1-\|\varphi(z)\|^{2}}=0$.

Then $C_{\varphi}: \mathcal{B}\left(B_{E}\right) \rightarrow \mathcal{B}\left(B_{E}\right)$ is a compact operator.

Proof. We are going to apply Lemma 4.4. Let $\left(f_{\alpha}\right)$ be a bounded net in $\mathcal{B}\left(B_{E}\right)$ converging to zero uniformly on compact sets. Recall that

$$
\mathcal{R}\left(f_{\alpha} \circ \varphi\right)(z)=\left\langle\nabla f_{\alpha}(\varphi(z)), \overline{\mathcal{R} \varphi(z)}\right\rangle .
$$

Let $\varepsilon>0$. By (ii) and (iii) there exists $\delta<1$ such that for $\|\varphi(z)\|>\delta$ we have

$$
\left(1-\|z\|^{2}\right) \frac{\sqrt{\left(1-\|\varphi(z)\|^{2}\right)\|\mathcal{R} \varphi(z)\|^{2}+|\langle\varphi(z), \mathcal{R} \varphi(z)\rangle|^{2}}}{1-\|\varphi(z)\|^{2}}<\varepsilon
$$

and hence using Lemma 2.1, we have

$$
\left(1-\|z\|^{2}\right)\left|\mathcal{R}\left(f_{\alpha} \circ \varphi\right)(z)\right| \leq \sup _{\alpha}\left\|\widetilde{\nabla} f_{\alpha}(\varphi(z))\right\| \varepsilon \leq \sup _{\alpha}\left\|f_{\alpha}\right\|_{i n v} \varepsilon .
$$

Denote $A_{\delta}=\left\{z \in B_{E}:\|\varphi(z)\| \leq \delta\right\}$. For $z \in A_{\delta}$ we use formula (4.15) obtained in the proof of Lemma 4.10 to have

$$
\begin{aligned}
& \left\langle\frac{\mathcal{R} \varphi(z)}{2\|\mathcal{R} \varphi(z)\|}, \overline{\nabla f(\varphi(z))}\right\rangle= \\
= & \frac{1}{s_{\varphi(z)}}\left(1-\frac{1}{s_{\varphi(z)}}\right) \frac{1}{2 \pi i} \int_{|\xi|=1} f\left(\varphi_{\varphi(z)}(\xi \varphi(z))\right) \frac{d \xi}{\xi^{2}} \frac{\langle\mathcal{R} \varphi(z), \varphi(z)\rangle}{2\|\mathcal{R} \varphi(z)\|\|\varphi(z)\|^{2}} \\
- & \frac{1}{s_{\varphi(z)}} \frac{1}{2 \pi i} \int_{|\xi|=1} f\left(\varphi_{\varphi(z)}\left(\xi \frac{\mathcal{R} \varphi(z)}{2\|\mathcal{R} \varphi(z)\|}\right)\right) \frac{d \xi}{\xi^{2}} .
\end{aligned}
$$

Hence for each $z \in A_{\delta}$,

$$
\begin{aligned}
& \left(1-\|z\|^{2}\right)\left|\left\langle\nabla f_{\alpha}(\varphi(z)), \overline{\mathcal{R} \varphi(z)}\right\rangle\right| \leq \\
& \frac{\left(1-\|z\|^{2}\right)}{\|\varphi(z)\|} \frac{1}{s_{\varphi(z)}}\left(\frac{1}{s_{\varphi(z)}}-1\right)\|\mathcal{R} \varphi(z)\| \int_{0}^{2 \pi}\left|f_{\alpha}\left(\varphi_{\varphi(z)}\left(e^{i t} \varphi(z)\right)\right)\right| \frac{d t}{2 \pi} \\
+ & \frac{2\left(1-\|z\|^{2}\right)}{s_{\varphi(z)}}\|\mathcal{R} \varphi(z)\| \int_{0}^{2 \pi}\left|f_{\alpha}\left(\varphi_{\varphi(z)}\left(e^{i t} \frac{\mathcal{R} \varphi(z)}{2\|\mathcal{R} \varphi(z)\|}\right)\right)\right| \frac{d t}{2 \pi} .
\end{aligned}
$$

Bearing in mind (2.12) in Lemma 2.2 and that $\lim _{\epsilon \rightarrow 0} \frac{1}{\epsilon}\left(\frac{1}{\sqrt{1-\epsilon^{2}}}-1\right)=0$, there is $C>0$ such that for $\|\varphi(z)\| \leq \delta$ we have

$$
\frac{\left(1-\|z\|^{2}\right)}{\|\varphi(z)\|} \frac{1}{s_{\varphi(z)}}\left(\frac{1}{s_{\varphi(z)}}-1\right)\|\mathcal{R} \varphi(z)\| \leq \frac{2}{\|\varphi(z)\|}\left(\frac{1}{\left(1-\|\varphi(z)\|^{2}\right)^{1 / 2}}-1\right) \leq C .
$$


In particular, for each $\delta<1$ there exists $C_{\delta}>0$ such that

$$
\begin{gathered}
\left(1-\|z\|^{2}\right)\left|\left\langle\nabla f_{\alpha}(\varphi(z)), \overline{\mathcal{R} \varphi(z)}\right\rangle\right| \leq \\
C_{\delta}\left(\int_{0}^{2 \pi}\left|f_{\alpha}\left(\varphi_{\varphi(z)}\left(e^{i t} \varphi(z)\right)\right) \frac{d t}{2 \pi}+\int_{0}^{2 \pi}\right| f_{\alpha}\left(\varphi_{\varphi(z)}\left(e^{i t} \frac{\mathcal{R} \varphi(z)}{2\|\mathcal{R} \varphi(z)\|}\right)\right) \mid \frac{d t}{2 \pi}\right)
\end{gathered}
$$

when $\|\varphi(z)\| \leq \delta$. Therefore, since

$$
\left\{\varphi_{\varphi(z)}(\xi \varphi(z)): \xi \in \mathbb{T}\right\} \cup\left\{\varphi_{\varphi(z)}\left(\xi \frac{\mathcal{R} \varphi(z)}{2\|\mathcal{R} \varphi(z)\|}\right): \xi \in \mathbb{T}\right\}
$$

is compact in $B_{E}$, we have for each $z \in A_{\delta}$ that

$$
\left(1-\|z\|^{2}\right)\left|\left\langle\nabla f_{\alpha}(\varphi(z)), \overline{\mathcal{R} \varphi(z)}\right\rangle\right| \rightarrow 0 .
$$

To finish the proof we use that $\varphi\left(A_{\delta}\right)$ is relatively compact in $B_{E}$. So, given $\varepsilon>0$ there exists a finite family of points $\left\{z_{k}: 1 \leq k \leq N\right\} \subset A_{\delta}$ such that for each $z \in A_{\delta}$ there exists $z_{k}$ for which $\left\|\varphi(z)-\varphi\left(z_{k}\right)\right\|<\varepsilon$.

Now bearing in mind (2.11) to observe that $s_{y}^{2}\|\mathcal{R} \varphi(y)\| \leq 1$, we may use Lemma 4.12 to have for each $z, z^{\prime} \in A_{\delta}$

$$
\begin{aligned}
& \mid\left\langle\nabla f_{\alpha}(\varphi(z)), s_{z}^{2} \overline{\mathcal{R} \varphi(z)}\right\rangle-\left\langle\nabla f_{\alpha}\left(\varphi\left(z^{\prime}\right)\right), s_{z^{\prime}}^{2} \overline{\left.\mathcal{R} \varphi\left(z^{\prime}\right)\right\rangle}\right| \leq C_{\delta}\left\|f_{\alpha}\right\|_{\mathcal{B}}\left\|\varphi(z)-\varphi\left(z^{\prime}\right)\right\| . \\
& \text { Hence } \sup _{z \in A_{\delta}}\left|\left\langle\nabla f_{\alpha}(\varphi(z)), s_{z}^{2} \overline{\mathcal{R} \varphi(z)}\right\rangle\right| \leq C^{\prime} \varepsilon+\max _{1 \leq k \leq n}\left|\left\langle\nabla f_{\alpha}\left(\varphi\left(z_{k}\right)\right), s_{z_{k}}^{2} \overline{\mathcal{R} \varphi\left(z_{k}\right)}\right\rangle\right| .
\end{aligned}
$$

The proof is then complete using (4.19).

Corollary 4.14. Assume that $\{\varphi(z):\|\varphi(z)\| \leq \delta\}$ is relatively compact for all $\delta<1$. Then $C_{\varphi}: \mathcal{B}\left(B_{E}\right) \rightarrow \mathcal{B}\left(B_{E}\right)$ is a compact operator if and only if

$$
\begin{aligned}
& \text { (i) } \lim _{\|\varphi(z)\| \rightarrow 1} \frac{\left(1-\|z\|^{2}\right)\|\mathcal{R} \varphi(z)\|}{\sqrt{1-\|\varphi(z)\|^{2}}}=0, \text { and } \\
& \text { (ii) } \lim _{\|\varphi(z)\| \rightarrow 1} \frac{\left(1-\|z\|^{2}\right)|\langle\varphi(z), \mathcal{R} \varphi(z)\rangle|}{1-\|\varphi(z)\|^{2}}=0 .
\end{aligned}
$$

Corollary 4.15. Assume that $\|\varphi\|_{\infty}<1$. The composition operator $C_{\varphi}$ is compact if $\varphi\left(B_{E}\right)$ is relatively compact.

Example 4.16. Let $\left\{e_{n}\right\}$ be a sequence in the given basis $\left\{e_{k}\right\}$. If $\left\{\varphi_{n}\right\}$ is a sequence in $H^{\infty}\left(B_{E}\right)$ such that $\sum_{n=1}^{\infty}\left\|\varphi_{n}\right\|_{\infty}^{2}<1$, then the mapping $\varphi(z):=\sum_{n} \varphi_{n}(z) e_{n}$ yields a compact composition operator $C_{\varphi}$ on $\mathcal{B}\left(B_{E}\right)$.

In particular for $\varphi_{n}(z)=\prod_{j=n}^{2 n}\left\langle z, e_{j}\right\rangle, C_{\varphi}$ is compact on $\mathcal{B}\left(B_{E}\right)$.

Proof. Note that $\sup _{\|z\|<1}\|\varphi(z)\|^{2} \leq\left(\sum_{n=1}^{\infty} \sup _{\|z\|<1}\left|\varphi_{n}(z)\right|^{2}\right)<1$. Moreover, $\varphi\left(B_{E}\right)$ is relatively compact since it lies inside the Hilbert cube given by the sequence $\left(\left\|\varphi_{n}\right\|_{\infty}\right)$. Now, apply Corollary 4.15 .

To verify the particular case we use the inequality between geometric and arithmetic means, namely

$$
\left|\varphi_{n}(z)\right|=\prod_{j=n}^{2 n}\left|\left\langle z, e_{j}\right\rangle\right| \leq\left(\frac{1}{n+1} \sum_{j=n}^{2 n}\left|\left\langle z, e_{j}\right\rangle\right|\right)^{n+1} \leq(n+1)^{-\frac{n+1}{2}}\|z\|,
$$

which produces the estimate $\sum_{n=1}^{\infty}\left\|\varphi_{n}\right\|_{\infty}^{2} \leq \sum_{n=1}^{\infty}(n+1)^{-(n+1)}<1$. 
Next, we introduce a class of symbols $\varphi$ that allows a characterization of the compactness of $C_{\varphi}$. We say that the analytic mapping $\varphi: B_{E} \rightarrow B_{E}$ belongs to $\mathcal{B}_{0}\left(B_{E}, B_{E}\right)$ if

$$
\lim _{\|z\| \rightarrow 1}\left(1-\|z\|^{2}\right)\|\mathcal{R} \varphi(z)\|=0 .
$$

In particular any map with bounded radial derivative satisfies (4.20). It is easy to produce examples of maps in $\mathcal{B}_{0}\left(B_{E}, B_{E}\right)$ :

Proposition 4.17. Let $\left\{e_{n}\right\}$ be a sequence in the given basis $\left\{e_{k}\right\}$. If $\left\{\varphi_{n}\right\}_{n} \subset \mathcal{B}\left(B_{E}\right)$ is such that

$$
\lim _{\|z\| \rightarrow 1}\left(1-\|z\|^{2}\right)\left|\mathcal{R} \varphi_{n}(z)\right|=0 \text { for all } n \in \mathbb{N} \text { and } \sum_{n=1}^{\infty}\left\|\varphi_{n}\right\|_{\mathcal{B}\left(B_{E}\right)}^{2}<\infty,
$$

then $\varphi(z)=\sum_{n=1}^{\infty} \varphi_{n}(z) e_{n} \in \mathcal{B}_{0}\left(B_{E}, B_{E}\right)$.

Proof. Given $\varepsilon>0$ there exist $N \in \mathbb{N}$ and $0<\delta_{j}<1$ for $j=1, \cdots, N$ such that

$$
\left(1-\|z\|^{2}\right)^{2}\|\mathcal{R} \varphi(z)\|^{2} \leq \sum_{n=1}^{N}\left(1-\|z\|^{2}\right)^{2}\left|\mathcal{R} \varphi_{n}(z)\right|^{2}+\varepsilon^{2} / 2
$$

and

$$
\left(1-\|z\|^{2}\right)\left|\mathcal{R} \varphi_{j}(z)\right|<\varepsilon / \sqrt{2 N}, \quad\|z\|>\delta_{j}, \quad j=1, \cdots, N
$$

Hence if $\|z\|>\max _{1 \leq j \leq N}\left\{\delta_{j}\right\}$, then $\left(1-\|z\|^{2}\right)\|\mathcal{R} \varphi(z)\|<\varepsilon$.

Proposition 4.18. Let $\varphi \in \mathcal{B}_{0}\left(B_{E}, B_{E}\right)$ with $\varphi(0)=0$. Then

$$
\begin{aligned}
(i) \limsup _{\|z\| \rightarrow 1} \frac{\left(1-\|z\|^{2}\right)\|\mathcal{R} \varphi(z)\|}{\sqrt{1-\|\varphi(z)\|^{2}}} & =\limsup _{\|\varphi(z)\| \rightarrow 1} \frac{\left(1-\|z\|^{2}\right)\|\mathcal{R} \varphi(z)\|}{\sqrt{1-\|\varphi(z)\|^{2}}}, \\
\text { (ii) } \limsup _{\|z\| \rightarrow 1} \frac{\left(1-\|z\|^{2}\right)|\langle\varphi(z), \mathcal{R} \varphi(z)\rangle|}{1-\|\varphi(z)\|^{2}} & =\limsup _{\|\varphi(z)\| \rightarrow 1} \frac{\left(1-\|z\|^{2}\right)|\langle\varphi(z), \mathcal{R} \varphi(z)\rangle|}{1-\|\varphi(z)\|^{2}} .
\end{aligned}
$$

Proof. In case $\|\varphi\|_{\infty}<1$, both right hand limits are null and both left hand limits vanish according to the assumption.

Since $\|\varphi(z)\| \leq\|z\|$ by Lemma 2.2, the limits on the right hand side are not greater than those on the left hand side. Now, in case $\|\varphi\|_{\infty}=1$, there is a sequence $\left(z_{n}\right) \subset$ $B_{E}$ such that $\left\|z_{n}\right\| \rightarrow 1$ and $\lim \sup _{\|z\| \rightarrow 1} \frac{\left(1-\|z\|^{2}\right)\|\mathcal{R} \varphi(z)\|}{\sqrt{1-\|\varphi(z)\|^{2}}}=\lim _{n} \frac{\left(1-\left\|z_{n}\right\|^{2}\right)\left\|\mathcal{R} \varphi\left(z_{n}\right)\right\|}{\sqrt{1-\left\|\varphi\left(z_{n}\right)\right\|^{2}}}$. From the bounded sequence $\left(\left\|\varphi\left(z_{n}\right)\right\|\right)$ we get a convergent subsequence that we denote the same. If $\lim _{n}\left\|\varphi\left(z_{n}\right)\right\|=1$, we have $\lim \sup _{\|\varphi(z)\| \rightarrow 1} \frac{\left(1-\|z\|^{2}\right)\|\mathcal{R} \varphi(z)\|}{\sqrt{1-\|\varphi(z)\|^{2}}} \geq \lim _{n} \frac{\left(1-\left\|z_{n}\right\|^{2}\right)\left\|\mathcal{R} \varphi\left(z_{n}\right)\right\|}{\sqrt{1-\left\|\varphi\left(z_{n}\right)\right\|^{2}}}$ that leads to the equality $(i)$, while if $\lim _{n}\left\|\varphi\left(z_{n}\right)\right\|<1$, then $\lim \sup _{\|z\| \rightarrow 1} \frac{\left(1-\|z\|^{2}\right)\|\mathcal{R} \varphi(z)\|}{\sqrt{1-\|\varphi(z)\|^{2}}}=0$, so $(i)$ holds as well. The analogous argument for $(i i)$.

In the following result we replace condition (i) in Theorem 4.13 by the weaker one given by (4.1) and conditions (4.2) and (4.3) by the stronger ones given by taking $\lim _{\|\varphi(z)\| \rightarrow 1}$ instead of $\lim _{\|z\| \rightarrow 1}$. Since the proof follows the same arguments as in Theorem 4.13, it will only be sketched. 
Proposition 4.19. Let $\varphi: B_{E} \rightarrow B_{E}$ be analytic with $\varphi(0)=0$. If $\varphi$ satisfies (4.1),

$$
\begin{aligned}
& \lim _{\|z\| \rightarrow 1} \frac{\left(1-\|z\|^{2}\right)\|\mathcal{R} \varphi(z)\|}{\sqrt{1-\|\varphi(z)\|^{2}}}=0, \text { and } \\
& \lim _{\|z\| \rightarrow 1} \frac{\left(1-\|z\|^{2}\right)|\langle\varphi(z), \mathcal{R} \varphi(z)\rangle|}{1-\|\varphi(z)\|^{2}}=0,
\end{aligned}
$$

then $C_{\varphi}$ is compact on $\mathcal{B}\left(B_{E}\right)$.

Proof. By Lemma 2.2, we have $\|\varphi(z)\| \leq\|z\|$. The analogous estimate to (4.18) holds for $\|z\|>\delta$.

In the remaining case $\|z\| \leq \delta$, also $\|\varphi(z)\| \leq \delta$ so the estimates in the proof of Theorem 4.13 hold, that is, if $\|z\| \leq \delta$, then

$$
\left(1-\|z\|^{2}\right)\left|\left\langle\nabla f_{\alpha}(\varphi(z)), \overline{\mathcal{R} \varphi(z)}\right\rangle\right| \rightarrow 0 .
$$

Now the final argument in the proof of Theorem 4.13 relies on the relative compactness of $\varphi(\{\|z\| \leq \delta\})$.

Let us mention that (4.21) implies that $\varphi \in \mathcal{B}_{0}\left(B_{E}, B_{E}\right)$ and that combining the necessary condition obtained in Theorem 4.5 and Proposition 4.18 we get the following:

Corollary 4.20. Let $\varphi \in \mathcal{B}_{0}\left(B_{E}, B_{E}\right)$ with $\varphi(0)=0$. Then $C_{\varphi}$ is compact in $\mathcal{B}\left(B_{E}\right)$ if and only if $\varphi$ satisfies (4.1), (4.21) and (4.22).

\section{EXAmples}

In this section we provide a number of examples to discuss the relations among the various conditions we have found above.

Example 5.1. Consider $\left(\xi_{n}\right) \subset B_{E}$ such that

$$
\sup _{\|z\| \leq 1} \sum_{n}\left|\left\langle z, \xi_{n}\right\rangle\right|^{2} \leq 1
$$

Define $\varphi_{n}(z)=\left\langle z, \xi_{n}\right\rangle$ and $\varphi(z)=\sum_{n} \varphi_{n}(z) e_{n}$, where $\left\{e_{n}\right\}$ is an orthonormal sequence in $E$.

Then $\varphi$ satisfies (4.2). In particular $\varphi \in \mathcal{B}_{0}\left(B_{E}, B_{E}\right)$.

Moreover if $\left(\xi_{n}\right)$ is an orthogonal system we have that

(i) $\varphi$ satisfies (4.3) whenever $\sup _{n}\left\|\xi_{n}\right\|<1$,

(ii) $\varphi$ fails (4.3) whenever there exists $n_{0}$ with $\left\|\xi_{n_{0}}\right\|=1$,

(iii) $\varphi\left(B_{E}\right)$ is relatively compact whenever $\sum_{n}\left\|\xi_{n}\right\|^{2}<\infty$, and

(iv) $\varphi$ fails (4.1) whenever $\lim \sup _{n \rightarrow \infty}\left\|\xi_{n}\right\|>0$.

Proof. Assumption (5.1) guarantees that $\varphi$ is analytic and maps $B_{E}$ to $B_{E}$. Since $\varphi(0)=0$, by Lemma 2.2 , we have $\|\varphi(z)\| \leq\|z\|$ for any $z \in B_{E}$. Notice that $\mathcal{R} \varphi(z)=\sum_{n} \mathcal{R} \varphi_{n}(z) e_{n}=$ $\varphi(z)$ and using that $\alpha \mapsto \frac{\alpha}{\sqrt{1-\alpha^{2}}}$ is increasing for $0<\alpha<1$, we have

$$
\frac{\left(1-\|z\|^{2}\right)\|\mathcal{R} \varphi(z)\|}{\sqrt{1-\|\varphi(z)\|^{2}}}=\frac{\left(1-\|z\|^{2}\right)\|\varphi(z)\|}{\sqrt{1-\|\varphi(z)\|^{2}}} \leq \sqrt{1-\|z\|^{2}} .
$$

In particular $\varphi$ satisfies (4.2). 
0 .

Since $\langle\varphi(z), \mathcal{R} \varphi(z)\rangle=\|\varphi(z)\|^{2}$, we get that $\varphi$ satisfies $(4.3)$ if and only if $\lim _{\|\varphi(z)\| \rightarrow 1} \frac{1-\|z\|^{2}}{1-\|\varphi(z)\|^{2}}=$

Assume now that $\left(\xi_{n}\right)$ is an orthogonal system. Hence

$$
\|\varphi(z)\|^{2}=\sum_{n}\left|\left\langle z, \xi_{n}\right\rangle\right|^{2} \leq \sup _{n}\left\|\xi_{n}\right\|^{2} \sum_{n}\left|\left\langle z, \frac{\xi_{n}}{\left\|\xi_{n}\right\|}\right\rangle\right|^{2} \leq \sup _{n}\left\|\xi_{n}\right\|^{2}\|z\|^{2} .
$$

Assuming $\sup _{n}\left\|\xi_{n}\right\|^{2}<1$ we have $\varphi\left(B_{E}\right) \subset \delta B_{E}$ for some $\delta<1$ and (4.3) trivially holds which shows (i).

Assume now that $\left\|\xi_{n_{0}}\right\|=1$. Selecting $z=\lambda \xi_{n_{0}}$ we have that $\varphi(z)=\lambda e_{n_{0}}$ and

$$
\lim _{\|\varphi(z)\| \rightarrow 1} \frac{1-\|z\|^{2}}{1-\|\varphi(z)\|^{2}}\|\varphi(z)\|^{2}=1 \text {. }
$$

This gives (ii).

Now (iii) follows using that $\left|\varphi_{n}(z)\right| \leq\left\|\xi_{n}\right\|$ for each $n$. Hence $\varphi\left(B_{E}\right)$ is contained in the Hilbert cube given by the sequence $\left(\left\|\xi_{n}\right\|\right)$.

Finally, to show (iv), assume $\lim \sup _{n \rightarrow \infty}\left\|\xi_{n}\right\|>0$. Hence there exist $\varepsilon>0$ and indices $m_{n}$ such that $\left\|\xi_{m_{n}}\right\| \geq \varepsilon$. For each $0<\delta<1$ we have $\varphi\left(\delta \frac{\xi_{n}}{\left\|\xi_{n}\right\|}\right)=\delta\left\|\xi_{n}\right\| e_{n}$. Hence $\left\{\delta\left\|\xi_{m_{n}}\right\| e_{m_{n}}\right.$ : $n \in \mathbb{N}\} \subset \varphi\left(\delta B_{E}\right)$ which gives that $\varphi\left(\delta B_{E}\right)$ is not relatively compact in $B_{E}$.

In [5] it was shown that $\varphi(z)=\sum_{n=1}^{\infty} z_{n}^{n} e_{n}$ satisfies (4.1). Such $\varphi$ is a particular choice in the following example.

Example 5.2. Let $\left\{e_{k}\right\}$ be an orthonormal sequence in $E$. Let $F_{k}: \mathbb{D} \rightarrow \mathbb{D}$ be a sequence of analytic functions such that $F_{k}(0)=0$. Define

$$
\varphi(z):=\sum_{k=1}^{\infty} F_{k}\left(\left\langle z, e_{k}\right\rangle\right) e_{k} .
$$

(i) If $\left\|F_{k}\right\|_{\infty}<1$ for all $k \in \mathbb{N}$, then $\varphi$ satisfies (4.7).

(ii) If $F_{k} \in \mathcal{B}_{0}$, the little Bloch space, and $\left\|F_{k}\right\|_{\infty}<1$ for all $k \in \mathbb{N}$, then $\varphi$ satisfies (4.9).

(iii) If there exists $n_{0} \in \mathbb{N}$ such that $C_{F_{n_{0}}}$ is non-compact on $\mathcal{B}$, then $\varphi$ fails (4.3).

(iv) If $\sup _{k}\left\|F_{k}\right\|_{\infty}<1$, then $\varphi$ satisfies $\varphi\left(B_{E}\right) \subset \delta B_{E}$ for some $0<\delta<1$. In particular $\varphi$ satisfies (4.2) and (4.3).

(v) If $\sum_{k}\left\|F_{k}\right\|_{\infty}^{2}<\infty$, then $\varphi\left(B_{E}\right)$ is relatively compact in $B_{E}$.

(vi) If $\sum_{k}\left\|F_{k}\right\|_{\mathcal{B}}^{2}<\infty$, then $\varphi$ satisfies (4.1).

Proof. Notice that since $\left|F_{k}(\lambda)\right| \leq|\lambda|$ we have that $\varphi$ maps $B_{E}$ into $B_{E}$. Actually one has

$$
\|\varphi(z)\|^{2}=\sum_{k=1}^{\infty}\left|F_{k}\left(\left\langle z, e_{k}\right\rangle\right)\right|^{2} \leq \sum_{k=1}^{\infty}\left\|F_{k}\right\|_{\infty}^{2}\left|\left\langle z, e_{k}\right\rangle\right|^{2} \leq\|z\|^{2} \text { and further, }
$$

$$
\left.\|\varphi(z)\| \leq \sup _{k}\left\|F_{k}\right\|_{\infty}\right)\|z\| .
$$

Since $\varphi^{\prime}(z)(u)=\sum_{k=1}^{\infty} F_{k}^{\prime}\left(\left\langle z, e_{k}\right\rangle\right)\left\langle u, e_{k}\right\rangle e_{k}$, then

$$
\mathcal{R} \varphi(z)=\sum_{k=1}^{\infty} F_{k}^{\prime}\left(\left\langle z, e_{k}\right\rangle\right)\left\langle z, e_{k}\right\rangle e_{k}, \text { so }
$$




$$
\langle\varphi(z), \mathcal{R} \varphi(z)\rangle=\sum_{k=1}^{\infty} F_{k}\left(\left\langle z, e_{k}\right\rangle\right) \overline{F_{k}^{\prime}\left(\left\langle z, e_{k}\right\rangle\right)\left\langle z, e_{k}\right\rangle} .
$$

Statement (i) follows since $\varphi_{k, l}(\lambda)=F_{k}(\lambda) \delta_{k, l}$ and $\left\|F_{k}\right\|_{\infty}<1$ implies compactness of $C_{F_{k}}$.

To verify (ii) notice that $\frac{\left|\mathcal{R} \varphi_{n}(z)\right|}{1-\left|\varphi_{n}(z)\right|^{2}}=\frac{\left|F_{n}^{\prime}\left(\left\langle z, e_{n}\right\rangle\right) \|\left\langle z, e_{n}\right\rangle\right|}{1-\left|F_{n}\left(\left\langle z, e_{n}\right\rangle\right)\right|^{2}} \quad$ from where we conclude

$$
\frac{\left(1-\|z\|^{2}\right)\left|\mathcal{R} \varphi_{n}(z)\right|}{1-\left|\varphi_{n}(z)\right|^{2}} \leq \frac{\left(1-\left|\left\langle z, e_{n}\right\rangle\right|^{2}\right)\left|F_{n}^{\prime}\left(\left\langle z, e_{n}\right\rangle\right)\right|}{1-\left\|F_{n}\right\|_{\infty}^{2}}
$$

that shows (4.9).

Concerning (iii): since $C_{F_{n_{0}}}$ is non-compact then by Theorem 2 in [7] there exists $\left(\lambda_{n}\right) \subset \mathbb{D}$ for which $\left|F_{n_{0}}\left(\lambda_{n}\right)\right| \rightarrow 1$ (in particular $\left|\lambda_{n}\right| \rightarrow 1$ ) and

$$
\lim _{n} \frac{\left(1-\left|\lambda_{n}\right|^{2}\right)\left|F_{n_{0}}^{\prime}\left(\lambda_{n}\right)\right|}{1-\left|F_{n_{0}}\left(\lambda_{n}\right)\right|^{2}} \neq 0
$$

Selecting the sequence $\xi_{n}=\lambda_{n} e_{n_{0}}$, we have $\left\|\varphi\left(\xi_{n}\right)\right\|^{2}=\left|F_{n_{0}}\left(\lambda_{n}\right)\right|^{2},\left\|\mathcal{R} \varphi\left(\xi_{n}\right)\right\|=\left|F_{n_{0}}^{\prime}\left(\lambda_{n}\right) \| \lambda_{n}\right|$ and $\left\langle\mathcal{R} \varphi\left(\xi_{n}\right), \varphi\left(\xi_{n}\right)\right\rangle=\overline{F_{n_{0}}\left(\lambda_{n}\right)} F_{n_{0}}^{\prime}\left(\lambda_{n}\right) \lambda_{n}$. Therefore $\varphi$ fails (4.3).

To check (iv), choose $\delta=\sup _{k}\left\|F_{k}\right\|_{\infty}$ and use (5.2).

Since $\varphi\left(B_{E}\right)$ is contained in the Hilbert cube given by the sequence $\left(\left\|F_{k}\right\|_{\infty}\right)$ it is relatively compact. Thus (v) holds.

Finally to show (vi) we use the estimate for analytic functions $F: \mathbb{D} \rightarrow \mathbb{D}$ with $F(0)=0$ given by $|F(\lambda)| \leq\|F\|_{\mathcal{B}} \beta(0, \lambda)$ to obtain that $\varphi\left(\delta B_{E}\right)$ is contained in the Hilbert cube given by the sequence $\left(\left\|F_{k}\right\|_{\mathcal{B}} \beta(0, \delta)\right)$. This gives (4.1).

Example 5.3. Let $\left\{e_{k}\right\}$ be an orthonormal sequence in $E$. Let us consider $\varphi(z)=\sum_{k} \varphi_{k}(z) e_{k}$ where

$$
\varphi_{k}(z)=\left\langle z, e_{k}\right\rangle^{k}
$$

Then $\varphi$ satisfies (4.1) and fails (4.8). In particular $C_{\varphi}$ is non-compact on $\mathcal{B}\left(B_{E}\right)$.

Proof. Notice that $\varphi(z) \in B_{E}$ for each $z \in B_{E}$ because

$$
\sum_{k=1}^{\infty}\left|\varphi_{k}(z)\right|^{2} \leq \sum_{k=1}^{\infty}\left|\left\langle z, e_{k}\right\rangle\right|^{2} \leq\|z\|^{2} .
$$

It is clear that $\mathcal{R} \varphi_{k}(z)=k \varphi_{k}(z)$.

To show (4.1) just observe that $\sup _{\|z\| \leq \delta}\left|\varphi_{k}(z)\right| \leq \delta^{k}$. Denote

$$
A_{k}=\sup _{z \in B_{E}} \frac{\left(1-\|z\|^{2}\right)\left|\mathcal{R} \varphi_{k}(z)\right|}{1-\left|\varphi_{k}(z)\right|^{2}}
$$

Let $z=\lambda e_{k}$ and estimate $A_{k} \geq \sup _{0<\lambda<1} \frac{\left(1-\lambda^{2}\right) k \lambda^{k}}{1-\lambda^{2 k}} \geq \sup _{k}\left(1-\frac{1}{k}\right)^{\frac{k}{2}}>0$. 
Example 5.4. Let $\left\{e_{k}\right\}$ be an orthonormal sequence in $E$. Let $\left(n_{k}\right)_{k \in \mathbb{N}}$ be an increasing sequence of natural numbers with $n_{0}=0$ and define $\varphi(z)=\sum_{k} \varphi_{k}(z) e_{k}$ and $\psi(z)=\sum_{k} \psi_{k}(z) e_{k}$ where

$$
\varphi_{k}(z)=\sum_{j=n_{k-1}+1}^{n_{k}} z_{j}^{2 k} \text { and } \psi_{k}(z)=\left(\sum_{j=n_{k-1}+1}^{n_{k}} z_{j}^{2}\right)^{k} .
$$

Then $\varphi$ and $\psi$ satisfy (4.1) but fail (4.7). Hence $C_{\varphi}$ and $C_{\psi}$ are non-compact on $\mathcal{B}\left(B_{E}\right)$.

Proof. Notice that $\varphi(z), \psi(z) \in B_{E}$ for each $z \in B_{E}$ because

$$
\max \left\{\left|\varphi_{k}(z)\right|^{2},\left|\psi_{k}(z)\right|^{2}\right\} \leq\left(\sum_{j=n_{k-1}+1}^{n_{k}}\left|z_{j}\right|^{2}\right)^{k} \leq \sum_{j=n_{k-1}+1}^{n_{k}}\left|z_{j}\right|^{2} .
$$

Condition (4.1) follows from the estimate $\max \left\{\left|\varphi_{k}(z)\right|^{2},\left|\psi_{k}(z)\right|^{2}\right\} \leq\|z\|^{2 k}$.

It is immediate to see that $\mathcal{R} \varphi_{k}(z)=2 k \varphi_{k}(z)$ and $\mathcal{R} \psi_{k}(z)=2 k \psi_{k}(z)$ and, for each $k, m \in \mathbb{N}$ we have

$$
\psi_{k, m}(\lambda)=\varphi_{k, m}(\lambda)=\lambda^{2 k}, \quad n_{k} \leq m \leq n_{k+1}
$$

and $\psi_{k, m}=\varphi_{k, m}=0$ otherwise.

We see that $C_{\varphi_{k, m}}$ is non-compact on $\mathcal{B}$ because

$$
\lim _{\left|\lambda^{2 k}\right| \rightarrow 1} \frac{\left(1-|\lambda|^{2}\right) 2 k|\lambda|^{2 k-1}}{1-|\lambda|^{4 k}} \neq 0
$$

due to the estimate $1-|\lambda|^{4 k} \leq 2 k\left(1-|\lambda|^{2}\right), \quad|\lambda|<1$.

\section{REFERENCES}

[1] O. Blasco, P. Galindo and A. Miralles, Bloch functions on the unit ball of an infinite dimensional Hilbert space, J. Func. Anal. 267 (2014), 1188-1204.

[2] O. Blasco, M. Lindström and J. Taskinen, Bloch-to-BMOA compositions in several complex variables, Complex Var. Theory Appl. 50 (2005), no. 14, 1061-1080.

[3] S. B. Chae, Holomorphy and calculus in normed spaces. With an appendix by Angus E. Taylor, Monographs and Textbooks in Pure and Applied Mathematics, 92. Marcel Dekker, Inc., New York, 1985. xii+421 pp.

[4] J. Dai, Compact composition operators on the Bloch space of the unit ball J. Math. Anal. Appl. 386 (2012), 294-299.

[5] D. García, M. Maestre and P. Sevilla-Peris, Composition operators between weighted spaces of holomorphic functions on Banach spaces, Ann. Acad. Sci. Fenn. Math. 29 (2004), 81-98.

[6] K. Goebel and S. Reich, Convexity, Hyperbolic Geometry, and Nonexpansive Mappings, Marcel Dekker, Inc., New York and Basel (1984).

[7] K. Madigan and A. Matheson, Compact composition operators on the Bloch space, Trans. Amer. Math. Soc. 347 (1995) 2679-2687.

[8] R. M. Timoney, Bloch functions in several complex variables I, Bull. London Math. Soc., 12 (1980) $241-267$.

[9] K. Zhu, Spaces of holomorphic functions in the unit ball, Grad. Texts in Math. 226, Springer Verlag 2005.

[10] K. Zhu, Operator theory in function spaces, Mathematical Surveys and Monographs 138, American Mathematical Society, Providence, RI (2007). 
Oscar Blasco. Departamento de Análisis Matemático, Universidad de Valencia, Valencia, SPAIN. e.MAIL: OSCAR.BLASCO@UV.ES

Pablo Galindo. Departamento de Análisis Matemático, Universidad de Valencia, Valencia, Spain. e.MAIL: PABLO.GALINDO@UV.ES

Mikael Lindström. Department of Mathematics, Abo Akademi University, Abo, Finland. e.MAIL: MLINDSTR@ABO.FI

Alejandro Miralles. Departament de Matemàtiques and Instituto Universitario de Matemáticas y Aplicaciones de Castellón (imAC), Universitat Jaume I de Castelló (UJi), Castelló, Spain. e.MAIL: MIRALLEA@UJI.ES 